\title{
Genetic Variants in Antineutrophil Cytoplasmic Antibody-Associated Vasculitis: A Bayesian Approach and Systematic Review
}

\author{
Kwang Seob Lee ${ }^{1,+}{ }^{,}$Andreas Kronbichler ${ }^{2,+}{ }^{+}$, Daniel Fernando Pereira Vasconcelos ${ }^{3}$, \\ Felipe Rodolfo Pereira da Silva ${ }^{3}{ }^{(D)}$, Younhee Ko ${ }^{4}$, Yeon $\mathrm{Su} \mathrm{Oh}^{5}$, Michael Eisenhut ${ }^{6}$, \\ Peter A. Merkel 7,8, David Jayne ${ }^{9,10}$, Christopher I. Amos ${ }^{11}$, Katherine A. Siminovitch ${ }^{12}$, \\ Chinar Rahmattulla ${ }^{13}$, Keum Hwa Lee ${ }^{14,15,16}$ (D) and Jae Il Shin 14,15,16,*(D) \\ 1 Severance Hospital, Yonsei University College of Medicine, Seoul 03722, Korea; kwangseob@yuhs.ac \\ 2 Department of Internal Medicine IV (Nephrology and Hypertension), Medical University Innsbruck, \\ 6020 Innsbruck, Austria; andreas.kronbichler@i-med.ac.at \\ 3 Laboratory of Histological Analysis and Preparation (LAPHIS), Federal University of Piaui, \\ Parnaiba 64202-020, Brazil; vasconcelos@ufpi.edu.br (D.F.P.V.); feliperodolfo.15@hotmail.com (F.R.P.d.S.) \\ 4 Division of Biomedical Engineering, Hankuk University of Foreign Studies, Gyeonggi-do 17035, Korea; \\ younko@hufs.ac.kr \\ 5 Yonsei University College of Medicine, Seoul 03722, Korea; nasaoh@naver.com \\ 6 Department of Pediatrics, Luton \& Dunstable University Hospital NHS Foundation Trust, \\ Luton LU4 0DZ, UK; michael_eisenhut@yahoo.com \\ 7 Division of Rheumatology, Department of Medicine, University of Pennsylvania, \\ Philadelphia, PA 19146, USA; Peter.Merkel@uphs.upenn.edu \\ 8 Department of Biostatistics, Epidemiology, and Informatics, University of Pennsylvania, \\ Philadelphia, PA 19146, USA \\ 9 Vasculitis and Lupus Clinic, Addenbrooke's Hospital, Cambridge CB2 0QQ, UK; dj106@cam.ac.uk \\ 10 Department of Medicine, University of Cambridge, Cambridge CB2 0QQ, UK \\ 11 Institute for Clinical and Translational Research, Baylor College of Medicine, Houston, TX 77030, USA; \\ Christopher.I.Amos@dartmouth.edu \\ 12 Mount Sinai Hospital, Lunenfeld-Tanenbaum Research Institute, Toronto General Research Institute and \\ University of Toronto, Toronto, ON M5G 1X5, Canada; Katherine.Siminovitch@sinaihealthsystem.ca \\ 13 Department of Pathology, Leiden University Medical Center, 2300 RC Leiden, The Netherlands; \\ c.rahmattulla@lumc.nl \\ 14 Department of Pediatrics, Yonsei University College of Medicine, Seoul 03722, Korea; AZSAGM@yuhs.ac \\ 15 Department of Pediatric Nephrology, Severance Children's Hospital, Seoul 03722, Korea \\ 16 Institute of Kidney Disease Research, Yonsei University College of Medicine, Seoul 03722, Korea \\ * Correspondence: SHINJI@yuhs.ac; Tel.: +82-2-2228-2050; Fax: +82-2-393-9118 \\ + These authors contributed equally to this work.
}

Received: 31 January 2019; Accepted: 19 February 2019; Published: 21 February 2019

check for updates Abstract: A number of genome-wide association studies (GWASs) and meta-analyses of genetic
variants have been performed in antineutrophil cytoplasmic antibody (ANCA)-associated vasculitis.
We reinterpreted previous studies using false-positive report probability (FPRP) and Bayesian false
discovery probability (BFDP). This study searched publications in PubMed and Excerpta Medica
Database (EMBASE) up to February 2018. Identification of noteworthy associations were analyzed
using FPRP and BFDP, and data (i.e., odds ratio (OR), 95\% confidence interval (CI), p-value) related to
significant associations were separately extracted. Using filtered gene variants, gene ontology (GO)
enrichment analysis and protein-protein interaction (PPI) networks were performed. Overall,
241 articles were identified, and 7 were selected for analysis. Single nucleotide polymorphisms (SNPs)
discovered by GWASs were shown to be noteworthy, whereas only 27\% of significant results from
meta-analyses of observational studies were noteworthy. Eighty-five percent of SNPs with borderline
-values $\left(5.0 \times 10^{-8}<p<0.05\right)$ in GWASs were found to be noteworthy. No overlapping SNPs 
were found between PR3-ANCA and MPO-ANCA vasculitis. GO analysis revealed immune-related GO terms, including "antigen processing and presentation of peptide or polysaccharide antigen via major histocompatibility complex (MHC) class II", "interferon-gamma-mediated (IFN- $\gamma$ ) signaling pathway". By using FPRP and BFDP, network analysis of noteworthy genetic variants discovered genetic risk factors associated with the IFN- $\gamma$ pathway as novel mechanisms potentially implicated in the complex pathogenesis of ANCA-associated vasculitis.

Keywords: antineutrophil cytoplasmic antibody (ANCA); proteinase 3; myeloperoxidase; single nucleotide polymorphism; vasculitis; meta-analysis; genome-wide association study

\section{Introduction}

Antineutrophil cytoplasmic antibody (ANCA)-associated vasculitis (AAV) is a group of autoimmune diseases characterized by the inflammation of small vessels. Clinical overlap among the different subtypes of AAV, granulomatosis with polyangiitis (GPA), microscopic polyangiitis (MPA) and eosinophilic granulomatosis with polyangiitis (EGPA) can result in diagnostic difficulties [1]. The clinical manifestations of the disease types can vary widely; most patients have signs of a lung, kidney, and/or ear, nose, and throat (ENT) involvement. ENT involvement is especially common in patients with GPA and EGPA [1]. A positive test for ANCA aids in the initial diagnosis of AAV. However, overlaps between disease phenotype and ANCA serotype limit the ability of the ANCA serotype to discriminate between the different disease phenotypes [2]. In general, a perinuclear ANCA (p-ANCA) - target antigen MPO-is found in $60-80 \%$ of MPA patients. On the other hand, cytoplasmic ANCA (c-ANCA) - target antigen proteinase 3 (PR3) - is frequently found in patients with severe GPA (approximately $90 \%$ of patients) and to a lesser degree in those with non-severe disease (approximately $50 \%$ of patients) [1].

Even though the exact pathogenesis of AAV is unclear, it is generally believed that AAV has a genetic background. Several genome-wide association studies (GWASs) and meta-analyses of observational studies employing single nucleotide polymorphisms (SNPs) revealed a number of genetic variants to be associated with AAV [3-6]. Up to date, there have been three GWASs and meta-analyses with replication cohorts: two by the US Vasculitis Clinical Research Consortium (VCRC) $[4,6]$ and one by the European Vasculitis Genetic Consortium (EVGC) [3]. Moreover, Rahmattulla and co-workers performed a meta-analysis with inclusion of EVGC GWAS data and other meta-analyses of observational studies [5].

Because the prior probabilities of genetic associations are low, the number of false-positive associations that are generated by chance alone is high. Not accounting for these low probabilities in the statistical analysis leads to an increased likelihood of finding false-positive associations. Since concerns have been raised about true positives of the discovered genetic variants in AAV, the aim of this study was to investigate whether these genetic variants are false-positives or are truly associated with AAV by applying a Bayesian approach. Moreover, we discussed and re-analyzed the filtered data based on the integration of the available results for genetic variants.

\section{Experimental Section}

\subsection{Method}

Database Search and Selection

The eligible studies were selected according to the standardized reporting protocol of systematic reviews and meta-analyses PRISMA (Preferred Reporting Items for Systematic reviews and Meta-Analyses) checklist (Table S1) [7]. A systematic search was performed in PubMed and EMBASE to retrieve studies published before 20 February 2018. Antineutrophil cytoplasmic antibody 
(ANCA)-associated vasculitis, polymorphisms, meta, genetic, variant and genome-wide association study (GWAS) were the terms used in the systematic search. At the end of the systematic search, 241 articles were identified, 13 were screened in detail, and 7 were selected for analysis [4-6,8-11]. (Figure 1 and Table S2).

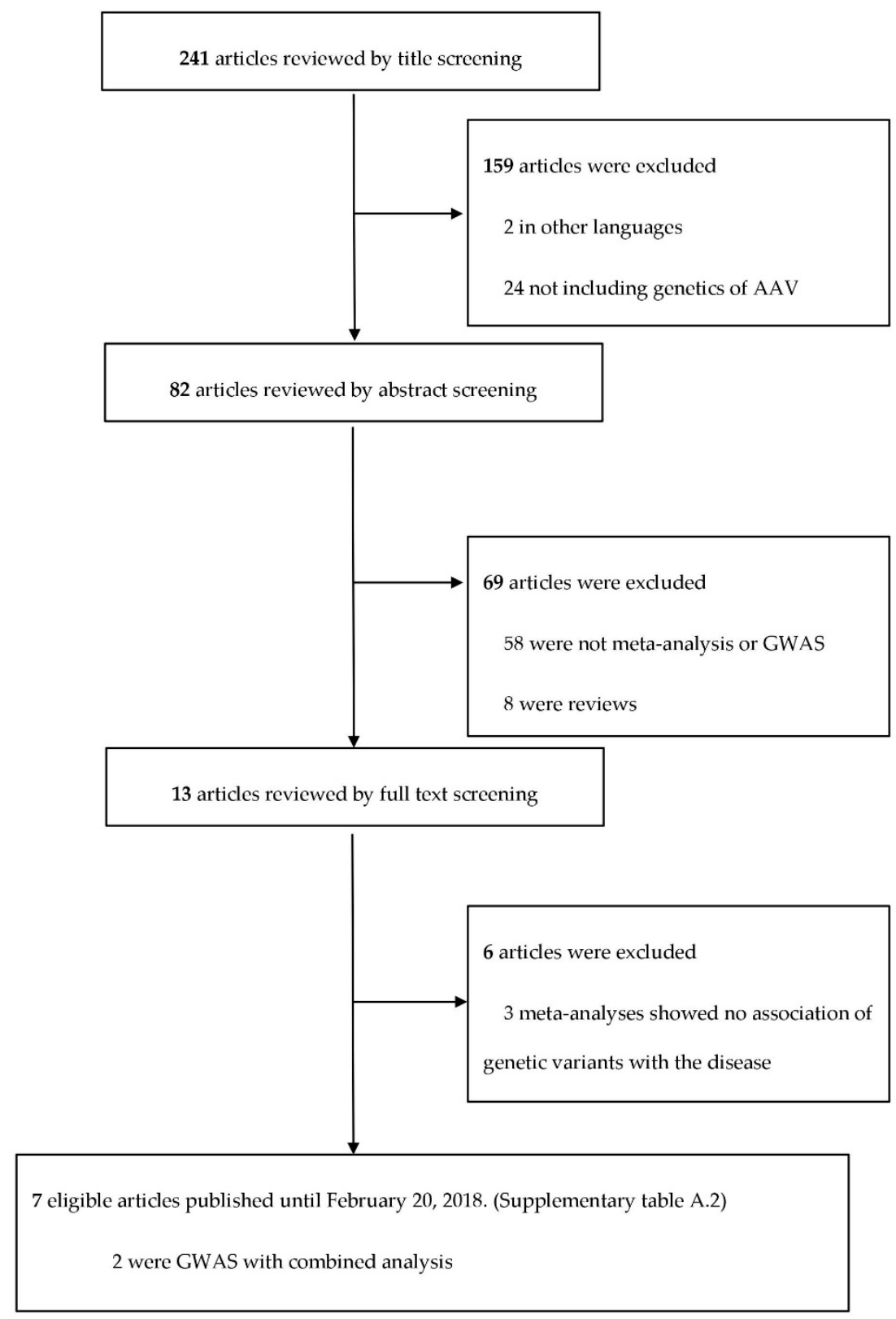

Figure 1. The process of the systematic search performed to study genetic variants in ANCA-associated vasculitis. Abbreviations used: AAV (ANCA-associated vasculitis), ANCA (anti-neutrophil cytoplasmic antibody), and GWAS (genome-wide association study).

The inclusion criteria were (1) a genetic meta-analysis or GWAS providing information of odds ratios (OR), 95\% confidence intervals (CI) in AAV (2) studies written in English, and (3) most recent larger meta-analyses (if smaller numbers have been analyzed before). The exclusion criteria were (1) studies not related to genetic polymorphisms or AAV, (2) articles not providing accurate data sets (i.e., review articles), and (3) older meta-analyses with overlapping genetic variants.

Data elements collected from meta-analyses included, when available, author, publication year, gene variant, single nucleotide polymorphism ID (rsID), genotype comparison, OR, 95\% CI, minor 
allelic frequency, ethnicity of study population, number of cases and controls, publication bias, and heterogeneity. Results of various subgroup analyses (i.e., Caucasian vs. mixed-population) were also extracted.

\subsection{Statistical Method}

Data related to statistically significant associations (i.e., OR, 95\% CI, $p$-value) and heterogeneity parameters (i.e., $p$-value and $I^{2}$ ) were separately extracted. Statistically significant associations were selected if the reported $p$-value was $<5 \times 10^{-8}$ for results of meta-analyses in GWASs for both the discovery and replication cohorts, and $<0.05$ for meta-analysis of general observational studies (i.e., case-control studies).

To assess and identify noteworthy associations, false-positive report probability (FPRP) and Bayesian false discovery probability (BFDP) were applied [12,13]. FPRP is defined as "the probability of no true association between a gene variant and disease (null hypothesis)" for a statistically significant association [12], and detailed calculation is presented in the supplementary file (Equation S1). FPRP is calculated with the observed p-value for the association, the statistical power of the test, and the prior probability that a molecular association is real. In this review, we calculated FPRP at two levels of assumed prior probabilities, which were $10^{-3}$ and $10^{-6}$. The interpretation for the noteworthiness of significant associations using FPRP is that the FPRP value of $<0.2$ is noteworthy [12].

BFDP is another Bayesian statistical method for detecting the true association between a gene variant and disease [13]. Main differences of BFDP compared to FPRP is that BFDP is independent from a statistical power and its approximation is based on a logistic regression model instead of a standard normal distribution [13]. The interpretation for the noteworthiness of significant associations using BFDP is that the BFDP value of $<0.8$ is noteworthy [13].

\subsection{Outcomes}

This study presents both FPRP and BFDP methods because the genetic epidemiologists and clinicians use BFDP less frequently than FPRP [12], although BFDP is a more recently developed method with a more substantial justification for its use [13]. By summarizing both results of FPRP and BFDP, we provide the readers options for interpreting noteworthiness.

\subsection{Construction of PPI (Protein-Protein Interaction) Network}

The STRING 9.1 network database is one of the largest databases of direct (physical) protein-protein interactions and indirect (functional) interactions constructed from various data sources including genomic context predictions, high-throughput experiments, co-expression, and known databases [14]. The STRING database covers 9.6 million proteins from more than 2031 organisms. In our study, we used STRING database to identify the PPIs associated with genes mapping to AAV, GPA, PR3-ANCA, and MPO-ANCA SNPs.

\section{Results}

\subsection{Computation of Noteworthy Variants}

The re-analysis of genetic variants was mainly driven by two categories of studies: one meta-analysis of observational studies and two GWASs with replication cohorts (combined analysis). All statistically significant SNPs and variants ( $p<0.05$ for observational studies and $p<5.0 \times 10^{-8}$ for GWASs) reported in the meta-analyses were included in this study. In addition, variants found in the GWAS meta-analysis with $5.0 \times 10^{-8}<p<0.05$, which were rejected as insignificant in their interpretation, were re-analyzed. Whether a variant was noteworthy was determined based on satisfaction of the condition in at least one of the values (FPRP $<0.2$ and BFDP $<0.8)$. The reported results were based on the various subtypes (GPA and MPA). In this study we followed the classification system used in the original studies. 
First, only $42 / 158$ (27\%) genetic comparisons in the meta-analysis of observational studies were noteworthy after re-analysis. In AAV, FPRP estimation resulted in 8/42 (19\%) and 3/42 (7\%) noteworthy genetic comparisons at the prior probability of $10^{-3}$ and $10^{-6}$ with the statistical power to detect an OR of 1.2. Likewise, 16/42 (38\%) and 12/42 (26\%) of comparisons were noteworthy at the prior probability of $10^{-3}$ and $10^{-6}$ with the statistical power to detect an OR of 1.5. BFDP estimation demonstrated $10 / 42(24 \%)$ and $6 / 42(14 \%)$ comparisons noteworthy at the prior probability of $10^{-3}$ and $10^{-6}$, respectively (Table 1 and Table S3).

In GPA, 4/50 (8\%) and 1/50 (2\%) comparisons were noteworthy in FPRP with the statistical power to detect an OR of 1.2 at the prior probability of $10^{-3}$ and $10^{-6} ; 9 / 50(18 \%), 3 / 50(6 \%)$ of comparisons with the statistical power to detect an OR of 1.5 at the prior probability of $10^{-3}$ and $10^{-6}$, respectively. In comparison, $15 / 50(30 \%)$ and 9/50 (18\%) variations were noteworthy in BFDP estimation at the prior probability of $10^{-3}$ and $10^{-6}$ (Table 1 and Table S3).

In PR3-ANCA, 5/24 (21\%) comparisons were noteworthy in FPRP while 10/24 (42\%) were found noteworthy in BFDP. Variants marked with 'NA' in the tables were not assessable with FPRP due to the mathematical error of calculating the inverse of the cumulative distribution, but BFDP was still computable. All the noteworthy variants in PR3-ANCA with FPRP estimation were also noteworthy in BFDP estimation (Table 2 and Table S4). No noteworthy comparison was observed in both MPA and MPO-ANCA vasculitis (Tables 1 and 2 and Tables S3 and S4).

Secondly, 22/28 (79\%) SNPs in the meta-analyses of GWASs were noteworthy in total. Without exception, statistically significant SNPs discovered by GWAS meta-analyses $\left(p<5.0 \times 10^{-8}\right)$ were also noteworthy in FPRP estimation at the prior probabilities of either $10^{-3}$ or $10^{-6}$ with the statistical power to detect an OR of 1.2 or 1.5 and in BFDP estimation at the probability of $10^{-3}$ or $10^{-6}$ except where mathematical errors did not allow FPRP. However, among the 14 SNPs with $p$-values ranging between 0.05 and $5.0 \times 10^{-8}$, which were considered as statistically non-significant in GWAS meta-analyses, 8 SNPs were reported noteworthy in both FPRP and BFDP estimation (Table 4 and Table S5).

We could re-analyze one GWAS of patients with GPA, while the remaining two GWASs were unable to be re-assessed due to the absence of $95 \%$ CI data. With our re-analyses, all the significant SNPs $\left(p<5.0 \times 10^{8}\right)$ observed in the GWASs were noteworthy in FPRP and BFDP estimation. However, among the borderline SNPs rejected by the GWAS with $p>5.0 \times 10^{-8}, 53 / 62(85 \%)$ of the SNPs were found noteworthy in our re-analysis (Table 4 and Table S6).

\subsection{Gene Network Analysis}

FPRP and BFDP computation reported all the SNPs with genome-wide significance discovered by GWASs as noteworthy, which may indicate that SNPs identified to be significant within GWASs are highly credible due to a conservative statistical standard and larger sample size. Based on this result, the SNPs' related protein-protein interaction (PPI) network of AAV subgroups and a Venn diagram of SNPs were constructed with the noteworthy genetic variants sorted by FPRP and BFDP and all the meaningful GWAS SNPs. The Venn diagram showed that no SNPs overlap between PR3-ANCA and MPO-ANCA vasculitis (Figure 2A and Figure S1). The PPI networks among genes mapping to PR3-ANCA and MPO-ANCA vasculitis-associated SNPs showed closer connections (Figure 2B,C). Interestingly, the genes associated with interferon-gamma (IFN- $\gamma$ )-mediated signaling pathway are highly enriched in both PR3-ANCA and MPO-ANCA vasculitis-associated SNPs (e.g., yellow nodes in the PPI networks). In addition, the MPA subtype was not included in the diagram, as no noteworthy SNPs have been reported in our analysis. The PPI network of AAV showed a variety of network connections. The major genes included in the network were major histocompatibility complex (MHC) class I and II genes (i.e., human leukocyte antigen (HLA)-A, -B, -C, -G, -F, -DPA1, -DPB1, -DQB1, -DRA). Other subtypes are shown in Figure 3 and Figure S2. 
Table 1. Meta-analysis results of observational studies; gene variants with statistical significance ( $p$-value $<0.05$ ), found to be noteworthy by false-positive report probability (FPRP) or Bayesian false discovery probability (BFDP) for each clinical diagnosis antineutrophil cytoplasmic antibody (ANCA)-associated vasculitis (AAV), granulomatosis with polyangiitis (GPA), and microscopic polyangiitis (MPA)).

\begin{tabular}{|c|c|c|c|c|c|c|c|c|c|c|c|c|c|c|c|c|c|c|}
\hline \multirow{3}{*}{$\begin{array}{l}\text { Gene/ } \\
\text { Variant }\end{array}$} & \multirow{3}{*}{$\begin{array}{l}\text { Minor } \\
\text { Allele/ } \\
\text { Comparison }\end{array}$} & \multirow{3}{*}{ OR $(95 \% \mathrm{CI})$} & \multirow{3}{*}{$\begin{array}{l}p \text {-Value for } \\
\text { Meta-Analysis }\end{array}$} & \multirow{3}{*}{$\begin{array}{l}\text { Publications } \\
\quad(n)\end{array}$} & \multirow{3}{*}{$\begin{array}{c}\text { Diagnosis } \\
\text { (Clinical } \\
\text { Subtypes) }\end{array}$} & \multirow{3}{*}{$\begin{array}{l}\text { No. of } \\
\text { Cases/ } \\
\text { Controls }\end{array}$} & \multirow{3}{*}{$I^{2}(\%)$} & \multirow{3}{*}{$I^{2}(\mathrm{P})$} & \multirow{3}{*}{$\begin{array}{l}\text { Egger's } \\
p \text {-Value }\end{array}$} & \multirow{3}{*}{$\begin{array}{l}\text { Power } \\
\text { OR } \\
1.2\end{array}$} & \multirow{3}{*}{$\begin{array}{l}\text { Power } \\
\text { OR } \\
1.5\end{array}$} & \multicolumn{4}{|c|}{ FPRP Values at Prior Probability } & \multirow{3}{*}{$\begin{array}{l}\text { BFDP } \\
0.001\end{array}$} & \multirow{3}{*}{$\begin{array}{l}\text { BFDP } \\
0.000001\end{array}$} & \multirow{3}{*}{ Author, Year } \\
\hline & & & & & & & & & & & & \multicolumn{2}{|c|}{ OR 1.2} & \multicolumn{2}{|c|}{ OR 1.5} & & & \\
\hline & & & & & & & & & & & & 0.001 & 0.000001 & 0.001 & 0.000001 & & & \\
\hline $\begin{array}{l}\text { CD226 } \\
\text { rs763361 }\end{array}$ & $\mathrm{T}$ & $\begin{array}{c}1.14 \\
(1.07-1.21)\end{array}$ & $<0.001$ & 3 & AAV & $2422 / 17898$ & 0 & 0.444 & 0.792 & 0.954 & 1.000 & 0.017 & 0.945 & 0.016 & 0.942 & 0.437 & 0.999 & $\begin{array}{c}\text { Rahmattulla, et al. } \\
2016 \text { [5] }\end{array}$ \\
\hline $\begin{array}{l}\text { CTLA-4 } \\
\text { rs3087243 } \\
\text { (CT60) }\end{array}$ & A & $\begin{array}{c}0.81 \\
(0.75-0.87)\end{array}$ & $<0.001$ & 3 & AAV & 2015/7855 & 25 & 0.262 & 0.122 & 0.218 & 1.000 & 0.000 & 0.033 & 0.000 & 0.007 & 0.001 & 0.347 & $\begin{array}{l}\text { Rahmattulla, et al. } \\
2016[5]\end{array}$ \\
\hline CTLA-4 & $(\mathrm{AT})_{86}$ & $\begin{array}{c}0.54 \\
(0.43-0.67)\end{array}$ & $<0.001$ & 4 & AAV & $303 / 543$ & 89 & $<0.001$ & 0.946 & 0.000 & 0.028 & 0.348 & 0.998 & 0.001 & 0.437 & 0.010 & 0.913 & $\begin{array}{c}\text { Rahmattulla, et al. } \\
2016 \text { [5] }\end{array}$ \\
\hline $\begin{array}{l}\text { CTLA-4 } \\
\text { rs3087243 } \\
\text { (CT60) }\end{array}$ & AA vs. GG & $\begin{array}{c}0.693 \\
(0.512-0.796)\end{array}$ & $6.39 \times 10^{-5}$ & 2 & AAV & 797/9669 & 57.7 & 0.124 & & 0.005 & 0.708 & 0.045 & 0.824 & 0.000 & 0.029 & 0.018 & 0.948 & Lee, et al. 2012 [8] \\
\hline $\begin{array}{l}\text { HLA-DPA1 } \\
\text { rs9277341 }\end{array}$ & C & $\begin{array}{c}0.35 \\
(0.30-0.40)\end{array}$ & $<0.001$ & 2 & AAV & $1032 / 2200$ & 54 & 0.116 & 0.215 & NA & NA & NA & NA & NA & NA & 0.000 & 0.000 & $\begin{array}{c}\text { Rahmattulla, et al. } \\
2016[5]\end{array}$ \\
\hline $\begin{array}{l}\text { HLA-DPB2 } \\
\text { rs3130215 }\end{array}$ & A & $\begin{array}{c}1.40 \\
(1.29-1.52)\end{array}$ & $<0.001$ & 3 & AAV & $1417 / 7249$ & 99 & $<0.001$ & 0.446 & 0.000 & 0.950 & 0.000 & 0.000 & 0.000 & 0.000 & 0.000 & 0.000 & $\begin{array}{c}\text { Rahmattulla, et al. } \\
2016 \text { [5] }\end{array}$ \\
\hline HLA-DRB4 & - & $\begin{array}{c}1.69 \\
(1.36-2.10)\end{array}$ & $<0.001$ & 4 & AAV & $260 / 1845$ & 61 & 0.055 & 0.533 & 0.001 & 0.141 & 0.686 & 1.000 & 0.015 & 0.940 & 0.259 & 0.997 & $\begin{array}{c}\text { Rahmattulla, et al. } \\
2016[5]\end{array}$ \\
\hline $\begin{array}{l}\text { HSD17B8 } \\
\text { rs421446 }\end{array}$ & C & $\begin{array}{c}0.40 \\
(0.34-0.48)\end{array}$ & $<0.001$ & 2 & $\mathrm{AAV}$ & 738/1872 & 0 & 0.620 & NA & NA & NA & NA & NA & NA & $\mathrm{NA}$ & 0.000 & 0.000 & $\begin{array}{c}\text { Rahmattulla, et al. } \\
2016 \text { [5] }\end{array}$ \\
\hline $\begin{array}{c}\text { IRF5 } \\
\text { rs10954213 }\end{array}$ & G & $\begin{array}{c}0.77 \\
(0.70-0.83)\end{array}$ & $<0.001$ & 3 & AAV & $1535 / 6977$ & 99 & $<0.001$ & 0.948 & 0.019 & 1.000 & 0.000 & 0.000 & 0.000 & 0.000 & 0.000 & 0.001 & $\begin{array}{c}\text { Rahmattulla, et al. } \\
2016[5]\end{array}$ \\
\hline $\begin{array}{l}\text { PTPN22 } \\
\text { rs2476601 }\end{array}$ & T vs. C & $\begin{array}{c}1.415 \\
(1.228-1.630)\end{array}$ & $1.59 \times 10^{-6}$ & 3 & AAV & $1184 / 10459$ & 0 & 0.393 & 0.481 & 0.011 & 0.791 & 0.119 & 0.931 & 0.002 & 0.160 & 0.091 & 0.990 & Lee, et al. 2012 [8] \\
\hline $\begin{array}{l}\text { PTPN22 } \\
\text { rs2476601 }\end{array}$ & A & $\begin{array}{c}1.39 \\
(1.24-1.56)\end{array}$ & $<0.001$ & 4 & AAV & $2099 / 8678$ & 0 & 0.693 & 0.500 & 0.006 & 0.902 & 0.004 & 0.780 & 0.000 & 0.024 & 0.002 & 0.654 & $\begin{array}{c}\text { Rahmattulla, et al. } \\
2016[5]\end{array}$ \\
\hline $\begin{array}{l}\text { RING1/RXRB } \\
\text { rs213213 }\end{array}$ & A & $\begin{array}{c}1.71 \\
(1.57-1.86)\end{array}$ & $<0.001$ & 3 & $\mathrm{AAV}$ & $1414 / 7238$ & 73 & 0.026 & 0.187 & NA & NA & NA & NA & NA & NA & 0.000 & 0.000 & $\begin{array}{c}\text { Rahmattulla, et al. } \\
2016[5]\end{array}$ \\
\hline $\begin{array}{l}\text { RXRB } \\
\text { rs6531 }\end{array}$ & C & $\begin{array}{c}1.63 \\
(1.50-1.77)\end{array}$ & $<0.001$ & 3 & $A A V$ & $1557 / 6955$ & 96 & $<0.001$ & 0.292 & NA & NA & NA & NA & NA & NA & 0.000 & 0.000 & $\begin{array}{l}\text { Rahmattulla, et al. } \\
2016 \text { [5] }\end{array}$ \\
\hline $\begin{array}{c}\text { RXRB } \\
\text { rs9277935 }\end{array}$ & $\mathrm{T}$ & $\begin{array}{c}0.44 \\
(0.37-0.50)\end{array}$ & $<0.001$ & 3 & AAV & $1417 / 7233$ & 73 & 0.025 & 0.393 & NA & NA & NA & NA & NA & NA & 0.000 & 0.000 & $\begin{array}{c}\text { Rahmattulla, et al. } \\
2016 \text { [5] }\end{array}$ \\
\hline SERPINA 1 & $Z$ allele & $\begin{array}{c}2.94 \\
(2.22-3.88)\end{array}$ & $<0.001$ & 8 & $\mathrm{AAV}$ & $3662 / 8581$ & 41 & 0.092 & 0.078 & 0.000 & 0.000 & 0.173 & 0.995 & 0.000 & 0.025 & 0.000 & 0.005 & $\begin{array}{c}\text { Rahmattulla, et al. } \\
2016 \text { [5] }\end{array}$ \\
\hline $\begin{array}{c}\text { TLR9 } \\
\text { rs352162 }\end{array}$ & $\mathrm{T}$ & $\begin{array}{c}1.58 \\
(1.43-1.75)\end{array}$ & $<0.001$ & 1 & AAV & $1289 / 1898$ & 96 & $<0.001$ & NA & NA & NA & NA & NA & NA & NA & 0.000 & 0.000 & $\begin{array}{l}\text { Rahmattulla, et al. } \\
2016 \text { [5] }\end{array}$ \\
\hline $\begin{array}{l}\text { PTPN22 } \\
\text { rs2476601 }\end{array}$ & T vs. C & $\begin{array}{c}2.042 \\
(1.534-2.719)\end{array}$ & $1.02 \times 10^{-6}$ & 2 & $\begin{array}{c}\text { ANCA (+) } \\
\text { GPA }\end{array}$ & - & 0 & 0.989 & NA & 0.000 & 0.017 & 0.882 & 0.999 & 0.056 & 0.855 & 0.375 & 0.998 & Lee, et al. 2012 [8] \\
\hline $\begin{array}{l}\text { CD226 } \\
\text { rs763361 }\end{array}$ & $\mathrm{T}$ & $\begin{array}{c}1.19 \\
(1.11-1.28)\end{array}$ & $<0.001$ & 3 & GPA & $2021 / 17898$ & 72.2 & 0.006 & & 0.589 & 1.000 & 0.005 & 0.832 & 0.003 & 0.745 & 0.124 & 0.993 & $\begin{array}{l}\text { Rahmattulla, et al. } \\
2016 \text { [5] }\end{array}$ \\
\hline $\begin{array}{c}\text { CLTA-4 } \\
\text { rs3087243 }\end{array}$ & A & $\begin{array}{c}0.80 \\
(0.73-0.87)\end{array}$ & $<0.001$ & 3 & GPA & $1561 / 7855$ & 38.7 & 0.180 & & 0.170 & 1.000 & 0.001 & 0.521 & 0.000 & 0.156 & 0.011 & 0.915 & $\begin{array}{c}\text { Rahmattulla, et al. } \\
2016 \text { [5] }\end{array}$ \\
\hline CTLA-4 & $(\mathrm{AT})_{86}$ & $\begin{array}{c}0.44 \\
(0.34-0.57)\end{array}$ & $<0.001$ & 3 & GPA & $210 / 432$ & 86.5 & 0.001 & & 0.000 & 0.001 & 0.434 & 0.999 & 0.001 & 0.381 & 0.002 & 0.670 & $\begin{array}{c}\text { Rahmattulla, et al. } \\
2016 \text { [5] }\end{array}$ \\
\hline
\end{tabular}


Table 1. Cont

\begin{tabular}{|c|c|c|c|c|c|c|c|c|c|c|c|c|c|c|c|c|c|c|}
\hline \multirow{3}{*}{$\begin{array}{l}\text { Gene/ } \\
\text { Variant }\end{array}$} & \multirow{3}{*}{$\begin{array}{c}\text { Minor } \\
\text { Allele/ } \\
\text { Comparison }\end{array}$} & \multirow{3}{*}{ OR $(95 \% \mathrm{CI})$} & \multirow{3}{*}{$\begin{array}{l}p \text {-Value for } \\
\text { Meta-Analysis }\end{array}$} & \multirow{3}{*}{$\begin{array}{l}\text { Publications } \\
\text { (n) }\end{array}$} & \multirow{3}{*}{$\begin{array}{l}\text { Diagnosis } \\
\text { (Clinical } \\
\text { Subtypes) }\end{array}$} & \multirow{3}{*}{$\begin{array}{l}\text { No. of } \\
\text { Cases/ } \\
\text { Controls }\end{array}$} & \multirow{3}{*}{$I^{2}(\%)$} & \multirow{3}{*}{$I^{2}(\mathrm{P})$} & \multirow{3}{*}{$\begin{array}{l}\text { Egger's } \\
p \text {-Value }\end{array}$} & \multirow{3}{*}{$\begin{array}{l}\text { Power } \\
\text { OR } \\
1.2\end{array}$} & \multirow{3}{*}{$\begin{array}{l}\text { Power } \\
\text { OR } \\
1.5\end{array}$} & \multicolumn{4}{|c|}{ FPRP Values at Prior Probability } & \multirow{3}{*}{$\begin{array}{l}\text { BFDP } \\
0.001\end{array}$} & \multirow{3}{*}{$\begin{array}{l}\text { BFDP } \\
0.000001\end{array}$} & \multirow{3}{*}{ Author, Year } \\
\hline & & & & & & & & & & & & \multicolumn{2}{|c|}{ OR 1.2} & \multicolumn{2}{|c|}{ OR 1.5} & & & \\
\hline & & & & & & & & & & & & 0.001 & 0.000001 & 10.001 & 0.000001 & & & \\
\hline $\begin{array}{c}\text { CTLA-4 } \\
\text { rs3087243 } \\
\text { (CT60) }\end{array}$ & A vs. G & $\begin{array}{c}0.79 \\
(0.70-0.89)\end{array}$ & $9.83 \times 10^{-5}$ & 2 & GPA & $880 / 1969$ & & & & 0.190 & 0.997 & 0.358 & 0.982 & 0.096 & 0.914 & 0.779 & 1.000 & $\begin{array}{c}\text { Chung, et al. } 2012 \\
\text { [10] }\end{array}$ \\
\hline $\begin{array}{l}\text { HLA-DPA1 } \\
\text { rs9277341 }\end{array}$ & C & $\begin{array}{c}0.35 \\
(0.30-0.41)\end{array}$ & $<0.001$ & 2 & GPA & $1032 / 2200$ & 54.8 & 0.109 & & NA & NA & NA & NA & NA & NA & 0.000 & 0.000 & $\begin{array}{c}\text { Rahmattulla, et al } \\
2016[5]\end{array}$ \\
\hline HLA-DPB1*0301 & - & $\begin{array}{c}0.23 \\
(0.16-0.32)\end{array}$ & $<0.001$ & 3 & GPA & $774 / 918$ & 61.7 & 0.050 & & NA & NA & NA & $\mathrm{NA}$ & NA & $\mathrm{NA}$ & 0.000 & 0.000 & $\begin{array}{c}\text { Rahmattulla, et al } \\
2016 \text { [5] }\end{array}$ \\
\hline HLA-DPB $1 * 0401$ & - & $\begin{array}{c}2.89 \\
(2.50-3.35)\end{array}$ & $<0.001$ & 3 & GPA & 774/918 & 67.5 & 0.026 & & NA & NA & NA & NA & NA & NA & 0.000 & 0.000 & $\begin{array}{c}\text { Rahmattulla, et al } \\
2016[5]\end{array}$ \\
\hline HLA-DR6 & - & $\begin{array}{c}0.45 \\
(0.33-0.62)\end{array}$ & $<0.001$ & 4 & GPA & $301 / 6132$ & 59.8 & 0.058 & & 0.000 & 0.008 & 0.927 & 1.000 & 0.114 & 0.992 & 0.511 & 0.999 & $\begin{array}{c}\text { Rahmattulla, et al } \\
2016[5]\end{array}$ \\
\hline $\begin{array}{c}\text { IRF5 } \\
\text { rs10954213 }\end{array}$ & G & $\begin{array}{c}0.66 \\
(0.59-0.74)\end{array}$ & $<0.001$ & 2 & GPA & $1021 / 6267$ & 99.1 & 0.000 & & 0.000 & 0.432 & 0.000 & 0.033 & 0.000 & 0.000 & 0.000 & 0.000 & $\begin{array}{c}\text { Rahmattulla, et al } \\
2016[5]\end{array}$ \\
\hline $\begin{array}{l}\text { PTPN22 } \\
\text { rs2476601 }\end{array}$ & A & $\begin{array}{c}1.43 \\
(1.26-1.62)\end{array}$ & $<0.001$ & 4 & GPA & $1616 / 8678$ & 0.0 & 0.411 & & 0.003 & 0.774 & 0.006 & 0.867 & 0.000 & 0.024 & 0.002 & 0.649 & $\begin{array}{c}\text { Rahmattulla, et al } \\
2016[5]\end{array}$ \\
\hline $\begin{array}{l}\text { RING1/RXRB } \\
\text { rs213213 }\end{array}$ & A & $\begin{array}{c}1.91 \\
(1.73-2.10)\end{array}$ & $<0.001$ & 3 & GPA & $1132 / 7238$ & 0.0 & 0.551 & & NA & NA & NA & NA & NA & NA & 0.000 & 0.000 & $\begin{array}{c}\text { Rahmattulla, et al } \\
2016[5]\end{array}$ \\
\hline $\begin{array}{l}\text { RXRB } \\
\text { rs6531 }\end{array}$ & C & $\begin{array}{c}1.70 \\
(1.55-1.86)\end{array}$ & $<0.001$ & 3 & GPA & $1211 / 6955$ & 96.5 & 0.000 & & NA & NA & NA & NA & NA & NA & 0.000 & 0.000 & $\begin{array}{c}\text { Rahmattulla, et al } \\
2016[5]\end{array}$ \\
\hline $\begin{array}{c}\text { RXRB } \\
\text { rs9277935 }\end{array}$ & $\mathrm{T}$ & $\begin{array}{c}0.37 \\
(0.31-0.43)\end{array}$ & $<0.001$ & 3 & GPA & $1135 / 7233$ & 0.0 & 0.798 & & NA & NA & NA & NA & NA & NA & 0.000 & 0.000 & $\begin{array}{c}\text { Rahmattulla, et al } \\
2016 \text { [5] }\end{array}$ \\
\hline SERPINA 1 & $Z$ allele & $\begin{array}{c}2.40 \\
(1.73-3.33)\end{array}$ & $<0.001$ & 4 & GPA & $972 / 2636$ & 0.0 & 0.763 & & 0.000 & 0.002 & 0.906 & 1.000 & 0.062 & 0.985 & 0.282 & 0.997 & $\begin{array}{c}\text { Rahmattulla, et al } \\
2016 \text { [5] }\end{array}$ \\
\hline HLA-DRB4 & - & $\begin{array}{c}2.06 \\
(1.57-2.69)\end{array}$ & $<0.001$ & 2 & EGPA & $150 / 691$ & 0.4 & 0.316 & & 0.000 & 0.010 & 0.754 & 1.000 & 0.011 & 0.918 & 0.089 & 0.990 & $\begin{array}{c}\text { Rahmattulla, et al } \\
2016[5]\end{array}$ \\
\hline
\end{tabular}

FPRP: false-positive report probability; BFDP: Bayesian false discovery probability; OR: odds ratio; $95 \%$ CI: 95\% confidence interval; No.: number; NA: not applicable. 
Table 2. Meta-analysis results of observational studies; gene variants with statistical significance ( $p$-value $<0.05$ ), found to be noteworthy by FPRP or BFDP for each serologic diagnosis (MPO-ANCA and PR3-ANCA).

\begin{tabular}{|c|c|c|c|c|c|c|c|c|c|c|c|c|c|c|c|c|c|c|}
\hline \multirow{3}{*}{$\begin{array}{l}\text { Gene/ } \\
\text { Variant }\end{array}$} & \multirow{3}{*}{$\begin{array}{l}\text { Minor } \\
\text { Allele/ } \\
\text { Comparison }\end{array}$} & \multirow{3}{*}{ OR $(95 \% \mathrm{CI})$} & \multirow{3}{*}{$\begin{array}{l}p \text {-Value for } \\
\text { Meta-Analysis }\end{array}$} & \multirow{3}{*}{$\begin{array}{l}\text { Publications } \\
\quad(n)\end{array}$} & \multirow{3}{*}{$\begin{array}{l}\text { Diagnosis } \\
\text { (Serologic } \\
\text { Subtypes) }\end{array}$} & \multirow{3}{*}{$\begin{array}{l}\text { No. of } \\
\text { Cases/ } \\
\text { Controls }\end{array}$} & \multirow{3}{*}{$\mathrm{I}^{2}(\%)$} & \multirow{3}{*}{$I^{2}(P)$} & \multirow{3}{*}{$\begin{array}{l}\text { Egger's } \\
p \text {-Value }\end{array}$} & \multirow{3}{*}{$\begin{array}{l}\text { Power } \\
\text { OR } \\
1.2\end{array}$} & \multirow{3}{*}{$\begin{array}{l}\text { Power } \\
\text { OR } \\
1.5\end{array}$} & \multicolumn{4}{|c|}{ FPRP Values at Prior Probability } & \multirow{3}{*}{$\begin{array}{c}\text { BFDP } \\
0.001\end{array}$} & \multirow{3}{*}{$\begin{array}{l}\text { BFDP } \\
0.000001\end{array}$} & \multirow{3}{*}{ Author, Year } \\
\hline & & & & & & & & & & & & \multicolumn{2}{|c|}{ OR 1.2} & \multicolumn{2}{|c|}{ OR 1.5} & & & \\
\hline & & & & & & & & & & & & 0.001 & 0.000001 & 0.001 & 0.000001 & & & \\
\hline $\begin{array}{l}\text { HLA-DPA1 } \\
\text { rs9277341 }\end{array}$ & C & $\begin{array}{c}0.27 \\
(0.22-0.33)\end{array}$ & $<0.001$ & 1 & PR3-ANCA & $578 / 1820$ & - & - & & NA & NA & NA & NA & NA & NA & 0.000 & 0.000 & $\begin{array}{l}\text { Rahmattulla, et al. } \\
2016 \text { [5] }\end{array}$ \\
\hline HLA-DPB1 ${ }^{*} 0401$ & - & $\begin{array}{c}3.93 \\
(2.75-5.62)\end{array}$ & $<0.001$ & 2 & PR3-ANCA & $183 / 139$ & 0.0 & 0.960 & & 0.000 & 0.000 & 0.615 & 0.999 & 0.001 & 0.495 & 0.000 & 0.170 & $\begin{array}{c}\text { Rahmattulla, et al. } \\
2016 \text { [5] }\end{array}$ \\
\hline $\begin{array}{l}\text { HLA-DPB2 } \\
\text { rs3130215 }\end{array}$ & A & $\begin{array}{c}0.65 \\
(0.55-0.77)\end{array}$ & $<0.001$ & 1 & PR3-ANCA & $326 / 5366$ & - & - & & 0.002 & 0.385 & 0.235 & 0.997 & 0.002 & 0.618 & 0.062 & 0.985 & $\begin{array}{c}\text { Rahmattulla, et al. } \\
2016 \text { [5] }\end{array}$ \\
\hline HLA-DRB1*15 & - & $\begin{array}{c}2.82 \\
(2.00-3.96)\end{array}$ & $<0.001$ & 2 & PR3-ANCA & $131 / 582$ & 84.0 & 0.002 & & 0.000 & 0.000 & 0.842 & 1.000 & 0.016 & 0.942 & 0.040 & 0.977 & $\begin{array}{c}\text { Rahmattulla, et al. } \\
2016 \text { [5] }\end{array}$ \\
\hline $\begin{array}{l}\text { RING1/RXRB } \\
\text { rs213213 }\end{array}$ & A & $\begin{array}{c}2.06 \\
(1.75-2.41)\end{array}$ & $<0.001$ & 1 & PR3-ANCA & $326 / 5366$ & - & - & & NA & NA & NA & NA & NA & NA & 0.000 & 0.000 & $\begin{array}{c}\text { Rahmattulla, et al. } \\
2016 \text { [5] }\end{array}$ \\
\hline $\begin{array}{l}\text { RXRB } \\
\text { rs6531 }\end{array}$ & C & $\begin{array}{c}2.19 \\
(1.92-2.51)\end{array}$ & $<0.001$ & 1 & PR3-ANCA & $478 / 5251$ & - & - & & NA & NA & NA & NA & NA & NA & 0.000 & 0.000 & $\begin{array}{c}\text { Rahmattulla, et al. } \\
2016[5]\end{array}$ \\
\hline $\begin{array}{c}\text { RXRB } \\
\text { rs } 9277935\end{array}$ & $\mathrm{~T}$ & $\begin{array}{c}0.24 \\
(0.17-0.33)\end{array}$ & $<0.001$ & 1 & PR3-ANCA & $326 / 5350$ & - & - & & NA & NA & NA & NA & NA & NA & 0.000 & 0.000 & $\begin{array}{c}\text { Rahmattulla, et al. } \\
2016[5]\end{array}$ \\
\hline SERPINA 1 & $\mathrm{Z}$ allele & $\begin{array}{c}3.53 \\
(2.28-5.49)\end{array}$ & $<0.001$ & 5 & PR3-ANCA & $280 / 4788$ & 21.3 & 0.279 & & 0.000 & 0.000 & 0.963 & 1.000 & 0.229 & 0.997 & 0.512 & 0.999 & $\begin{array}{c}\text { Rahmattulla, et al. } \\
2016 \text { [5] }\end{array}$ \\
\hline $\begin{array}{l}\text { TLR9 } \\
\text { rs352140 }\end{array}$ & $\mathrm{T}$ & $\begin{array}{c}1.28 \\
(1.12-1.45)\end{array}$ & 0.018 & 1 & PR3-ANCA & NR/NR & 0.0 & 0.782 & & 0.155 & 0.994 & 0.402 & 0.999 & 0.095 & 0.991 & 0.778 & 1.000 & $\begin{array}{c}\text { Rahmattulla, et al. } \\
2016[5]\end{array}$ \\
\hline $\begin{array}{c}\text { TLR9 } \\
\text { rs352162 }\end{array}$ & $\mathrm{T}$ & $\begin{array}{c}1.30 \\
(1.14-1.47)\end{array}$ & $<0.001$ & 1 & PR3-ANCA & NR/NR & 0.0 & 0.503 & & 0.101 & 0.989 & 0.221 & 0.996 & 0.028 & 0.967 & 0.532 & 0.999 & $\begin{array}{c}\text { Rahmattulla, et al. } \\
2016 \text { [5] }\end{array}$ \\
\hline
\end{tabular}


Table 3. Results of meta analyses (combined analysis) with genome-wide association studies and replication cohort. Noteworthy genetic variants with satisfied FPRP or BFDP values are presented. SNPs: single nucleotide polymorphism.

\begin{tabular}{|c|c|c|c|c|c|c|c|c|c|c|c|c|c|c|c|}
\hline \multirow{3}{*}{ Gene/ Variant } & \multirow{3}{*}{ Comparison } & \multirow{3}{*}{ OR $(95 \% \mathrm{CI})$} & \multirow{3}{*}{$p$-Value } & \multirow{3}{*}{$\begin{array}{l}\text { Diagnosis } \\
\text { (Clinical/ } \\
\text { Serologic } \\
\text { Subtypes) }\end{array}$} & \multirow{3}{*}{ Ethnicity } & \multirow{3}{*}{$\begin{array}{l}\text { No. of } \\
\text { Cases/ } \\
\text { Controls }\end{array}$} & \multirow{3}{*}{$\begin{array}{l}\text { Power } \\
\text { OR } 1.2\end{array}$} & \multirow{3}{*}{$\begin{array}{l}\text { Power } \\
\text { OR } 1.5\end{array}$} & \multicolumn{4}{|c|}{ FPRP Values at Prior Probability } & \multirow{3}{*}{$\begin{array}{c}\text { BFDP } \\
0.001\end{array}$} & \multirow{3}{*}{$\begin{array}{l}\text { BFDP } \\
0.000001\end{array}$} & \multirow{3}{*}{ Author, Year } \\
\hline & & & & & & & & & \multicolumn{2}{|c|}{ OR 1.2} & \multicolumn{2}{|c|}{ OR 1.5} & & & \\
\hline & & & & & & & & & 0.001 & 0.000001 & 0.001 & 0.000001 & & & \\
\hline \multicolumn{16}{|c|}{ SNPs Statistically Significant $\left(p<5.00 \times 10^{-8}\right)$} \\
\hline $\begin{array}{l}\text { HLA-DPA1 } \\
\text { rs9277341 }\end{array}$ & T vs. C & $2.44(2.21-2.69)$ & $6.09 \times 10^{-71}$ & AAV & Caucasian & $1986 / 4723$ & NA & NA & NA & NA & NA & NA & 0.000 & 0.000 & Merkel, et al. 2017 [4] \\
\hline $\begin{array}{l}\text { HLA-DPB1 } \\
\text { rs1042169 }\end{array}$ & G vs. A & $2.82(2.54-3.13)$ & $1.12 \times 10^{-84}$ & AAV & Caucasian & $1986 / 4723$ & NA & NA & NA & NA & NA & NA & 0.000 & 0.000 & Merkel, et al. 2017 [4] \\
\hline $\begin{array}{l}\text { HLA-DPB1 } \\
\text { rs141530233 }\end{array}$ & A del & $2.99(2.69-3.33)$ & $1.13 \times 10^{-89}$ & AAV & Caucasian & $1986 / 4723$ & NA & NA & NA & NA & NA & NA & 0.000 & 0.000 & Merkel, et al. 2017 [4] \\
\hline $\begin{array}{l}\text { HLA-DQA1 } \\
\text { rs35242582 }\end{array}$ & A vs. G & $1.60(1.46-1.76)$ & $6.34 \times 10^{-23}$ & AAV & Caucasian & $1986 / 4723$ & NA & NA & NA & NA & NA & NA & 0.000 & 0.000 & Merkel, et al. 2017 [4] \\
\hline $\begin{array}{l}\text { HLA-DQB1 } \\
\text { rs1049072 }\end{array}$ & A vs. G & $1.40(1.28-1.53)$ & $6.46 \times 10^{-13}$ & AAV & Caucasian & $1986 / 4723$ & 0.000 & 0.936 & 0.000 & 0.000 & 0.000 & 0.000 & 0.000 & 0.000 & Merkel, et al. 2017 [4] \\
\hline PRTN3 rs62132293 & G vs. C & $1.29(1.19-1.39)$ & $8.60 \times 10^{-11}$ & AAV & Caucasian & $1986 / 4723$ & 0.029 & 1.000 & 0.000 & 0.000 & 0.000 & 0.000 & 0.000 & 0.002 & Merkel, et al. 2017 [4] \\
\hline PTPN22 rs6679677 & A vs. C & $1.40(1.25-1.57)$ & $1.88 \times 10^{-8}$ & AAV & Caucasian & $1986 / 4723$ & 0.004 & 0.881 & 0.002 & 0.172 & 0.000 & 0.001 & 0.001 & 0.447 & Merkel, et al. 2017 [4] \\
\hline $\begin{array}{l}\text { SERPINA1 } \\
\text { rs28929474 }\end{array}$ & T vs. C & $2.18(1.75-2.71)$ & $3.09 \times 10^{-12}$ & AAV & Caucasian & $1986 / 4723$ & 0.000 & 0.000 & 0.056 & 0.855 & 0.000 & 0.001 & 0.000 & 0.010 & Merkel, et al. 2017 [4] \\
\hline $\begin{array}{l}\text { HLA-DPA1 } \\
\text { rs9277341 }\end{array}$ & C vs. T & $0.33(0.28-0.39)$ & $2.18 \times 10^{-39}$ & GPA & Caucasian & $750 / 1820$ & NA & NA & NA & NA & NA & NA & 0.000 & 0.000 & Xie, et al. 2013 [6] \\
\hline $\begin{array}{c}\text { HLA-DPB1 } \\
\text { rs9277554 }\end{array}$ & T vs. C & $0.24(0.20-0.30)$ & $1.92 \times 10^{-50}$ & GPA & Caucasian & $750 / 1820$ & NA & NA & NA & NA & NA & NA & 0.000 & 0.000 & Xie, et al. 2013 [6] \\
\hline SEMA6A rs26595 & Cvs. T & $0.74(0.67-0.82)$ & $2.09 \times 10^{-8}$ & GPA & Caucasian & $987 / 2731$ & 0.012 & 0.977 & 0.001 & 0.071 & 0.000 & 0.001 & 0.001 & 0.423 & Xie, et al. 2013 [6] \\
\hline $\begin{array}{c}\text { HLA-DQA2 } \\
\text { rs3998159 }\end{array}$ & C vs. A & $2.72(2.24-3.22)$ & $5.24 \times 10^{-25}$ & MPO-ANCA & Caucasian & $378 / 4723$ & NA & NA & NA & NA & NA & NA & 0.000 & 0.000 & Merkel, et al. 2017 [4] \\
\hline $\begin{array}{l}\text { HLA-DQA2 } \\
\text { rs7454108 }\end{array}$ & C vs. T & $2.73(2.25-3.24)$ & $5.03 \times 10^{-25}$ & MPO-ANCA & Caucasian & $378 / 4723$ & NA & NA & NA & NA & NA & NA & 0.000 & 0.000 & Merkel, et al. 2017 [4] \\
\hline $\begin{array}{l}\text { HLA-DQB1 } \\
\text { rs1049072 }\end{array}$ & A vs. G & $2.37(2.01-2,78)$ & $2.13 \times 10^{-24}$ & MPO-ANCA & Caucasian & $378 / 4723$ & NA & NA & NA & NA & NA & NA & 0.000 & 0.000 & Merkel, et al. 2017 [4] \\
\hline \multicolumn{16}{|c|}{ SNPs with Statistically Borderline Significance $\left(5.00 \times 10^{-8} \leq p<0.05\right)$} \\
\hline $\begin{array}{l}\text { PTPN22(R620W) } \\
\text { rs2476601 }\end{array}$ & A vs. G & $1.36(1.21-1.53)$ & $1.86 \times 10^{-7}$ & AAV & Caucasian & $1986 / 4723$ & 0.019 & 0.948 & 0.016 & 0.625 & 0.000 & 0.032 & 0.020 & 0.953 & Merkel, et al. 2017 [4] \\
\hline CCDC86 rs595018 & A vs. G & $1.46(1.27-1.69)$ & $1.60 \times 10^{-7}$ & GPA & Caucasian & $1986 / 4723$ & 0.004 & 0.641 & 0.084 & 0.902 & 0.001 & 0.058 & 0.033 & 0.971 & Xie, et al. 2013 [6] \\
\hline COBL rs1949829 & T vs. C & $1.78(1.42-2.24)$ & $4.19 \times 10^{-7}$ & GPA & Caucasian & $1986 / 4723$ & 0.000 & 0.072 & 0.694 & 0.996 & 0.012 & 0.549 & 0.177 & 0.995 & Xie, et al. 2013 [6] \\
\hline DCTD rs4862110 & C vs. T & $1.44(1.24-1.67)$ & $2.14 \times 10^{-6}$ & GPA & Caucasian & $1986 / 4723$ & 0.008 & 0.705 & 0.151 & 0.947 & 0.002 & 0.167 & 0.092 & 0.990 & Xie, et al. 2013 [6] \\
\hline DOK4 rs6023640 & T vs. G & $1.29(1.14-1.45)$ & $2.73 \times 10^{-5}$ & GPA & Caucasian & $987 / 2731$ & 0.113 & 0.994 & 0.148 & 0.946 & 0.019 & 0.664 & 0.445 & 0.999 & Xie, et al. 2013 [6] \\
\hline $\begin{array}{l}\text { FLJ34870 } \\
\text { rs7585252 }\end{array}$ & G vs. A & $1.26(1.13-1.40)$ & $1.74 \times 10^{-5}$ & GPA & Caucasian & $987 / 2731$ & 0.182 & 0.999 & 0.086 & 0.904 & 0.017 & 0.632 & 0.408 & 0.999 & Xie, et al. 2013 [6] \\
\hline PAEP rs705669 & G vs. A & $0.77(0.68-0.87)$ & $2.52 \times 10^{-5}$ & GPA & Caucasian & $987 / 2731$ & 0.102 & 0.990 & 0.210 & 0.964 & 0.027 & 0.733 & 0.520 & 0.999 & Xie, et al. 2013 [6] \\
\hline WSCD1 rs7503953 & A vs. C & $1.50(1.29-1.76)$ & $1.93 \times 10^{-7}$ & GPA & Caucasian & $1986 / 4723$ & 0.003 & 0.500 & 0.176 & 0.955 & 0.001 & 0.117 & 0.058 & 0.984 & Xie, et al. 2013 [6] \\
\hline
\end{tabular}


Table 4. Re-analysis of the SNPs discovered in genome-wide association studies of patients with GPA. Noteworthy genetic variants with satisfied FPRP or BFDP values are presented. Xie et al. only provided GPA SNPs with odds ratios (ORs) and 95\% confidence intervals (CIs).

\begin{tabular}{|c|c|c|c|c|c|c|c|c|c|c|c|c|c|c|c|}
\hline \multirow{3}{*}{ Gene/Variant } & \multirow{3}{*}{ Comparison } & \multirow{3}{*}{ OR $(95 \% \mathrm{CI})$} & \multirow{3}{*}{$p$-Value } & \multirow{3}{*}{$\begin{array}{l}\text { Diagnosis } \\
\text { (Clinical/ } \\
\text { Serologic } \\
\text { Subtypes) }\end{array}$} & \multirow{3}{*}{ Ethnicity } & \multirow{3}{*}{$\begin{array}{c}\text { No. of } \\
\text { Cases/ } \\
\text { Controls }\end{array}$} & \multirow{3}{*}{$\begin{array}{l}\text { Power } \\
\text { OR } 1.2\end{array}$} & \multirow{3}{*}{$\begin{array}{l}\text { Power } \\
\text { OR } 1.5\end{array}$} & \multicolumn{4}{|c|}{ FPRP Values at Prior Probability } & \multirow{3}{*}{$\begin{array}{c}\text { BFDP } \\
0.001\end{array}$} & \multirow{3}{*}{$\begin{array}{c}\text { BFDP } \\
0.000001\end{array}$} & \multirow{3}{*}{ Author, Year } \\
\hline & & & & & & & & & \multicolumn{2}{|c|}{ OR 1.2} & \multicolumn{2}{|c|}{ OR 1.5} & & & \\
\hline & & & & & & & & & 0.001 & 0.000001 & 0.001 & 0.000001 & & & \\
\hline \multicolumn{16}{|c|}{ SNPs with $p$-Value $<5.00 \times 10^{-8}$} \\
\hline $\begin{array}{l}\text { HLA-DOA } \\
\text { rs3130604 }\end{array}$ & G vs. A & $1.67(1.39-2.02)$ & $4.39 \times 10^{-8}$ & GPA & Caucasian & $459 / 1503$ & 0.000 & 0.134 & 0.277 & 0.997 & 0.001 & 0.487 & 0.025 & 0.962 & Xie, et al. 2013 [6] \\
\hline $\begin{array}{l}\text { HLA-DOA } \\
\text { rs763469 }\end{array}$ & A vs. G & $1.70(1.41-2.04)$ & $1.46 \times 10^{-8}$ & GPA & Caucasian & $459 / 1503$ & 0.000 & 0.089 & 0.114 & 0.992 & 0.000 & 0.116 & 0.003 & 0.764 & Xie, et al. 2013 [6] \\
\hline $\begin{array}{c}\text { HLA-DPA1 } \\
\text { rs2395309 }\end{array}$ & G vs. A & $0.27(0.20-0.36)$ & $2.15 \times 10^{-19}$ & GPA & Caucasian & $459 / 1503$ & NA & NA & NA & NA & NA & NA & 0.000 & 0.000 & Xie, et al. 2013 [6] \\
\hline HLA-DPA1 rs3077 & C vs. T & $0.27(0.20-0.36)$ & $2.68 \times 10^{-19}$ & GPA & Caucasian & $459 / 1503$ & NA & NA & NA & NA & NA & NA & 0.000 & 0.000 & Xie, et al. 2013 [6] \\
\hline $\begin{array}{c}\text { HLA-DPA1 } \\
\text { rs2301226 }\end{array}$ & T vs. C & $0.48(0.36-0.62)$ & $4.85 \times 10^{-8}$ & GPA & Caucasian & $459 / 1503$ & 0.000 & 0.006 & 0.613 & 0.999 & 0.003 & 0.762 & 0.023 & 0.959 & Xie, et al. 2013 [6] \\
\hline $\begin{array}{l}\text { HLA-DPA1 } \\
\text { rs9277341 }\end{array}$ & C vs. T & $0.30(0.25-0.38)$ & $1.84 \times 10^{-30}$ & GPA & Caucasian & $459 / 1503$ & NA & NA & NA & NA & NA & NA & 0.000 & 0.000 & Xie, et al. 2013 [6] \\
\hline $\begin{array}{l}\text { HLA-DPB1 } \\
\text { rs987870 }\end{array}$ & C vs. T & $0.26(0.19-0.37)$ & $6.09 \times 10^{-16}$ & GPA & Caucasian & $459 / 1503$ & 0.000 & 0.000 & 0.597 & 0.999 & 0.001 & 0.462 & 0.000 & 0.155 & Xie, et al. 2013 [6] \\
\hline $\begin{array}{l}\text { HLA-DPB1 } \\
\text { rs9277535 }\end{array}$ & G vs. A & $0.24(0.19-0.32)$ & $2.12 \times 10^{-28}$ & GPA & Caucasian & $459 / 1503$ & NA & NA & NA & NA & NA & NA & 0.000 & 0.000 & Xie, et al. 2013 [6] \\
\hline $\begin{array}{l}\text { HLA-DPB1 } \\
\text { rs9277554 }\end{array}$ & T vs. C & $0.22(0.17-0.28)$ & $4.88 \times 10^{-38}$ & GPA & Caucasian & $459 / 1503$ & NA & NA & NA & NA & NA & NA & 0.000 & 0.000 & Xie, et al. 2013 [6] \\
\hline $\begin{array}{l}\text { HLA-DPB1 } \\
\text { rs9277565 }\end{array}$ & T vs. C & $0.24(0.18-0.32)$ & $1.91 \times 10^{-24}$ & GPA & Caucasian & $459 / 1503$ & NA & NA & NA & NA & NA & NA & 0.000 & 0.000 & Xie, et al. 2013 [6] \\
\hline $\begin{array}{l}\text { HLA-DPB1 } \\
\text { rs2281389 }\end{array}$ & C vs. T & $0.24(0.18-0.34)$ & $1.69 \times 10^{-20}$ & GPA & Caucasian & $459 / 1503$ & 0.000 & 0.000 & 0.458 & 0.999 & 0.000 & 0.187 & 0.000 & 0.013 & Xie, et al. 2013 [6] \\
\hline $\begin{array}{l}\text { HLA-DPB1 } \\
\text { rs3128917 }\end{array}$ & G vs. T & $0.22(0.17-0.29)$ & $4.92 \times 10^{-33}$ & GPA & Caucasian & $459 / 1503$ & NA & NA & NA & NA & NA & NA & 0.000 & 0.000 & Xie, et al. 2013 [6] \\
\hline $\begin{array}{l}\text { HLA-DPB1 } \\
\text { rs3117222 }\end{array}$ & A vs. G & $0.22(0.17-0.29)$ & $3.05 \times 10^{-33}$ & GPA & Caucasian & $459 / 1503$ & NA & NA & NA & NA & NA & NA & 0.000 & 0.000 & Xie, et al. 2013 [6] \\
\hline $\begin{array}{c}\text { HLA-DPB2 } \\
\text { rs2064478 }\end{array}$ & A vs. $G$ & $0.22(0.17-0.30)$ & $4.29 \times 10^{-29}$ & GPA & Caucasian & $459 / 1503$ & NA & NA & NA & NA & NA & NA & 0.000 & 0.000 & Xie, et al. 2013 [6] \\
\hline $\begin{array}{l}\text { HLA-DPB2 } \\
\text { rs3130215 }\end{array}$ & A vs. G & $2.42(2.08-2.82)$ & $2.37 \times 10^{-30}$ & GPA & Caucasian & $459 / 1503$ & NA & NA & NA & NA & NA & NA & 0.000 & 0.000 & Xie, et al. 2013 [6] \\
\hline $\begin{array}{l}\text { HLA-DPB2 } \\
\text { rs3117230 }\end{array}$ & C vs. T & $0.22(0.17-0.30)$ & $4.29 \times 10^{-29}$ & GPA & Caucasian & $459 / 1503$ & NA & NA & NA & NA & NA & NA & 0.000 & 0.000 & Xie, et al. 2013 [6] \\
\hline $\begin{array}{l}\text { HLA-DPB2 } \\
\text { rs1883414 }\end{array}$ & T vs. C & $0.53(0.44-0.64)$ & $1.13 \times 10^{-11}$ & GPA & Caucasian & $459 / 1503$ & 0.000 & 0.009 & 0.031 & 0.970 & 0.000 & 0.005 & 0.000 & 0.039 & Xie, et al. 2013 [6] \\
\hline $\begin{array}{c}\text { HLA-DPB2 } \\
\text { rs4713607 }\end{array}$ & A vs. G & $0.60(0.52-0.70)$ & $6.70 \times 10^{-11}$ & GPA & Caucasian & $459 / 1503$ & 0.000 & 0.090 & 0.006 & 0.849 & 0.000 & 0.001 & 0.000 & 0.027 & Xie, et al. 2013 [6] \\
\hline $\begin{array}{l}\text { HLA-DPB2 } \\
\text { rs3129274 }\end{array}$ & G vs. A & $1.56(1.34-1.82)$ & $1.35 \times 10^{-8}$ & GPA & Caucasian & $459 / 1503$ & 0.000 & 0.309 & 0.036 & 0.974 & 0.000 & 0.048 & 0.002 & 0.708 & Xie, et al. 2013 [6] \\
\hline $\begin{array}{c}\text { HLA-DPB2 } \\
\text { rs3117016 }\end{array}$ & T vs. C & $0.48(0.41-0.57)$ & $1.09 \times 10^{-17}$ & GPA & Caucasian & $459 / 1503$ & NA & NA & NA & NA & NA & NA & 0.000 & 0.000 & Xie, et al. 2013 [6] \\
\hline $\begin{array}{l}\text { HLA-DPB2 } \\
\text { rs3117008 }\end{array}$ & T vs. C & $0.60(0.51-0.70)$ & $4.90 \times 10^{-11}$ & GPA & Caucasian & $459 / 1503$ & 0.000 & 0.090 & 0.006 & 0.849 & 0.000 & 0.001 & 0.000 & 0.027 & Xie, et al. 2013 [6] \\
\hline $\begin{array}{c}\text { HLA-DPB2 } \\
\text { rs3117004 }\end{array}$ & C vs. T & $0.57(0.48-0.68)$ & $1.90 \times 10^{-10}$ & GPA & Caucasian & $459 / 1503$ & 0.000 & 0.041 & 0.034 & 0.972 & 0.000 & 0.010 & 0.000 & 0.161 & Xie, et al. 2013 [6] \\
\hline $\begin{array}{l}\text { HLA-DPB2 } \\
\text { rs6901221 }\end{array}$ & Cvs. A & $0.42(0.32-0.55)$ & $6.08 \times 10^{-11}$ & GPA & Caucasian & $459 / 1503$ & 0.000 & 0.000 & 0.475 & 0.999 & 0.001 & 0.423 & 0.002 & 0.651 & Xie, et al. 2013 [6] \\
\hline
\end{tabular}


Table 4. Cont.

\begin{tabular}{|c|c|c|c|c|c|c|c|c|c|c|c|c|c|c|c|}
\hline \multirow{3}{*}{ Gene/ Variant } & \multirow{3}{*}{ Comparison } & \multirow{3}{*}{ OR $(95 \% \mathrm{CI})$} & \multirow{3}{*}{$p$-Value } & \multirow{3}{*}{$\begin{array}{l}\text { Diagnosis } \\
\text { (Clinical/ } \\
\text { Serologic } \\
\text { Subtypes) }\end{array}$} & \multirow{3}{*}{ Ethnicity } & \multirow{3}{*}{$\begin{array}{l}\text { No. of } \\
\text { Cases/ } \\
\text { Controls }\end{array}$} & \multirow{3}{*}{$\begin{array}{l}\text { Power } \\
\text { OR } 1.2\end{array}$} & \multirow{3}{*}{$\begin{array}{l}\text { Power } \\
\text { OR } 1.5\end{array}$} & \multicolumn{4}{|c|}{ FPRP Values at Prior Probability } & \multirow{3}{*}{$\begin{array}{c}\text { BFDP } \\
0.001\end{array}$} & \multirow{3}{*}{$\begin{array}{l}\text { BFDP } \\
0.000001\end{array}$} & \multirow{3}{*}{ Author, Year } \\
\hline & & & & & & & & & \multicolumn{2}{|c|}{ OR 1.2} & \multicolumn{2}{|c|}{ OR 1.5} & & & \\
\hline & & & & & & & & & 0.001 & 0.000001 & 0.001 & 0.000001 & & & \\
\hline COL11A2 rs986521 & C vs. T & $1.85(1.57-2.16)$ & $2.91 \times 10^{-14}$ & GPA & Caucasian & $459 / 1503$ & 0.000 & 0.004 & 0.000 & 0.246 & 0.000 & 0.000 & 0.000 & 0.000 & Xie, et al. 2013 [6] \\
\hline $\begin{array}{l}\text { COL11A2 } \\
\text { rs2855430 }\end{array}$ & T vs. C & $0.33(0.24-0.45)$ & $3.28 \times 10^{-13}$ & GPA & Caucasian & $459 / 1503$ & 0.000 & 0.000 & 0.505 & 0.999 & 0.001 & 0.357 & 0.000 & 0.237 & Xie, et al. 2013 [6] \\
\hline $\begin{array}{l}\text { COL11A2 } \\
\text { rs2855425 }\end{array}$ & C vs. T & $1.80(1.54-2.11)$ & $7.77 \times 10^{-14}$ & GPA & Caucasian & $459 / 1503$ & 0.000 & 0.012 & 0.001 & 0.594 & 0.000 & 0.000 & 0.000 & 0.000 & Xie, et al. 2013 [6] \\
\hline $\begin{array}{l}\text { COL11A2 } \\
\text { rs2855459 }\end{array}$ & T vs. C & $0.32(0.23-0.44)$ & $2.14 \times 10^{-13}$ & GPA & Caucasian & $459 / 1503$ & 0.000 & 0.000 & 0.548 & 0.999 & 0.001 & 0.427 & 0.000 & 0.287 & Xie, et al. 2013 [6] \\
\hline RXRB rs6531 & Cvs. T & $1.80(1.54-2.11)$ & $8.48 \times 10^{-14}$ & GPA & Caucasian & $459 / 1503$ & 0.000 & 0.012 & 0.001 & 0.594 & 0.000 & 0.000 & 0.000 & 0.000 & Xie, et al. 2013 [6] \\
\hline HSD17B8 rs439205 & T vs. C & $0.31(0.24-0.39)$ & $3.51 \times 10^{-23}$ & GPA & Caucasian & $459 / 1503$ & NA & NA & NA & NA & NA & NA & 0.000 & 0.000 & Xie, et al. 2013 [6] \\
\hline HSD17B8 rs421446 & C vs. T & $0.39(0.31-0.48)$ & $8.90 \times 10^{-20}$ & GPA & Caucasian & $459 / 1503$ & NA & NA & NA & NA & NA & NA & 0.000 & 0.000 & Xie, et al. 2013 [6] \\
\hline RING1 rs213213 & A vs. G & $1.83(1.57-2.14)$ & $6.98 \times 10^{-15}$ & GPA & Caucasian & $459 / 1503$ & 0.000 & 0.006 & 0.001 & 0.375 & 0.000 & 0.000 & 0.000 & 0.000 & Xie, et al. 2013 [6] \\
\hline RING1 rs213212 & G vs. T & $1.85(1.58-2.17)$ & $7.63 \times 10^{-15}$ & GPA & Caucasian & $459 / 1503$ & 0.000 & 0.005 & 0.001 & 0.439 & 0.000 & 0.000 & 0.000 & 0.000 & Xie, et al. 2013 [6] \\
\hline COBL rs1949829 & T vs. C & $2.19(1.68-2.86)$ & $3.58 \times 10^{-9}$ & GPA & Caucasian & $459 / 1503$ & 0.000 & 0.003 & 0.632 & 0.999 & 0.003 & 0.759 & 0.017 & 0.946 & Xie, et al. 2013 [6] \\
\hline CCDC86 rs595018 & A vs. G & $1.61(1.36-1.90)$ & $2.74 \times 10^{-8}$ & GPA & Caucasian & $459 / 1503$ & 0.000 & 0.201 & 0.064 & 0.986 & 0.000 & 0.080 & 0.003 & 0.764 & Xie, et al. 2013 [6] \\
\hline WSCD1 rs7503953 & A vs. C & $1.72(1.44-2.06)$ & $1.39 \times 10^{-9}$ & GPA & Caucasian & $459 / 1503$ & 0.000 & 0.068 & 0.076 & 0.988 & 0.000 & 0.053 & 0.001 & 0.555 & Xie, et al. 2013 [6] \\
\hline \multicolumn{16}{|c|}{ SNPs Reported Non-Significant $\left(5.00 \times 10^{-8} \leq p<1.00 \times 10^{-4}\right)$} \\
\hline TCEB3 rs2076346 & C vs. T & $1.46(1.24-1.73)$ & $8.62 \times 10^{-6}$ & GPA & Caucasian & $459 / 1503$ & 0.012 & 0.623 & 0.512 & 0.999 & 0.019 & 0.952 & 0.433 & 0.999 & Xie, et al. 2013 [6] \\
\hline DAB1 rs264036 & C vs. T & $0.72(0.62-0.85)$ & $9.82 \times 10^{-5}$ & GPA & Caucasian & $459 / 1503$ & 0.042 & 0.818 & 0.713 & 1.000 & 0.113 & 0.992 & 0.807 & 1.000 & Xie, et al. 2013 [6] \\
\hline DAB1 rs542873 & T vs. C & $1.37(1.18-1.59)$ & $4.70 \times 10^{-5}$ & GPA & Caucasian & $459 / 1503$ & 0.041 & 0.884 & 0.457 & 0.999 & 0.037 & 0.975 & 0.601 & 0.999 & Xie, et al. 2013 [6] \\
\hline DAB1 rs197644 & G vs. A & $1.37(1.18-1.59)$ & $3.89 \times 10^{-5}$ & GPA & Caucasian & $459 / 1503$ & 0.041 & 0.884 & 0.457 & 0.999 & 0.037 & 0.975 & 0.601 & 0.999 & Xie, et al. 2013 [6] \\
\hline LPHN2 rs11579502 & Cvs. T & $1.56(1.28-1.90)$ & $9.43 \times 10^{-6}$ & GPA & Caucasian & $459 / 1503$ & 0.005 & 0.348 & 0.684 & 1.000 & 0.027 & 0.966 & 0.458 & 0.999 & Xie, et al. 2013 [6] \\
\hline $\begin{array}{l}\text { NCKAP5 } \\
\text { rs1134119 }\end{array}$ & C vs. T & $1.79(1.38-2.32)$ & $8.96 \times 10^{-6}$ & GPA & Caucasian & $459 / 1503$ & 0.001 & 0.091 & 0.896 & 1.000 & 0.106 & 0.992 & 0.658 & 0.999 & Xie, et al. 2013 [6] \\
\hline $\begin{array}{l}\text { NCKAP5 } \\
\text { rs7585252 }\end{array}$ & G vs. A & $1.35(1.16-1.57)$ & $9.69 \times 10^{-5}$ & GPA & Caucasian & $459 / 1503$ & 0.063 & 0.914 & 0.607 & 0.999 & 0.096 & 0.991 & 0.785 & 1.000 & Xie, et al. 2013 [6] \\
\hline $\begin{array}{c}\text { NEK10 rs1579900 } \\
\text { NeK10 ro }\end{array}$ & T vs. G & $1.51(1.24-1.85)$ & $3.97 \times 10^{-5}$ & GPA & Caucasian & $459 / 1503$ & 0.013 & 0.474 & 0.840 & 1.000 & 0.128 & 0.993 & 0.800 & 1.000 & Xie, et al. 2013 [6] \\
\hline $\begin{array}{l}\text { CTNNB1 } \\
\text { rs } 9842536\end{array}$ & T vs. C & $1.46(1.23-1.74)$ & $1.63 \times 10^{-5}$ & GPA & Caucasian & $459 / 1503$ & 0.014 & 0.619 & 0.624 & 0.999 & 0.037 & 0.974 & 0.575 & 0.999 & Xie, et al. 2013 [6] \\
\hline C3orf58 rs1512779 & Cvs. A & $0.72(0.61-0.84)$ & $2.86 \times 10^{-5}$ & GPA & Caucasian & $459 / 1503$ & 0.032 & 0.836 & 0.484 & 0.999 & 0.034 & 0.972 & 0.579 & 0.999 & Xie, et al. 2013 [6] \\
\hline PLSCR4 rs7628805 & A vs. C & $1.41(1.18-1.67)$ & $9.72 \times 10^{-5}$ & GPA & Caucasian & $459 / 1503$ & 0.031 & 0.763 & 0.691 & 1.000 & 0.083 & 0.989 & 0.754 & 1.000 & Xie, et al. 2013 [6] \\
\hline $\begin{array}{c}\text { ST6GAL1 } \\
\text { rs10513807 }\end{array}$ & G vs. A & $0.71(0.61-0.83)$ & $9.49 \times 10^{-6}$ & GPA & Caucasian & $459 / 1503$ & 0.022 & 0.785 & 0.436 & 0.999 & 0.021 & 0.956 & 0.471 & 0.999 & Xie, et al. 2013 [6] \\
\hline $\begin{array}{l}\text { KIAA0746 } \\
\text { rs4269167 }\end{array}$ & T vs. C & $0.73(0.63-0.86)$ & $7.72 \times 10^{-5}$ & GPA & Caucasian & $459 / 1503$ & 0.057 & 0.861 & 0.747 & 1.000 & 0.163 & 0.995 & 0.858 & 1.000 & Xie, et al. 2013 [6] \\
\hline DCTD rs4862110 & C vs. T & $1.63(1.36-1.94)$ & $5.00 \times 10^{-8}$ & GPA & Caucasian & $459 / 1503$ & 0.000 & 0.175 & 0.118 & 0.993 & 0.000 & 0.178 & 0.007 & 0.878 & Xie, et al. 2013 [6] \\
\hline OSMR rs357291 & C vs. A & $0.72(0.62-0.84)$ & $2.81 \times 10^{-5}$ & GPA & Caucasian & $459 / 1503$ & 0.032 & 0.836 & 0.484 & 0.999 & 0.034 & 0.972 & 0.579 & 0.999 & Xie, et al. 2013 [6] \\
\hline SEMA6A rs26595 & C vs. T & $0.74(0.63-0.86)$ & $9.58 \times 10^{-5}$ & GPA & Caucasian & $459 / 1503$ & 0.061 & 0.913 & 0.586 & 0.999 & 0.086 & 0.989 & 0.766 & 1.000 & Xie, et al. 2013 [6] \\
\hline GRIA1 rs10515687 & T vs. C & $1.56(1.25-1.94)$ & $8.11 \times 10^{-5}$ & GPA & Caucasian & $459 / 1503$ & 0.009 & 0.362 & 0.874 & 1.000 & 0.150 & 0.994 & 0.811 & 1.000 & Xie, et al. 2013 [6] \\
\hline WWC1 rs3853242 & G vs. A & $0.74(0.63-0.86)$ & $8.55 \times 10^{-5}$ & GPA & Caucasian & $459 / 1503$ & 0.061 & 0.913 & 0.586 & 0.999 & 0.086 & 0.989 & 0.766 & 1.000 & Xie, et al. 2013 [6] \\
\hline ERGIC1 rs1564259 & A vs. G & $0.69(0.58-0.83)$ & $7.19 \times 10^{-5}$ & GPA & Caucasian & $459 / 1503$ & 0.023 & 0.642 & 0.785 & 1.000 & 0.114 & 0.992 & 0.797 & 1.000 & Xie, et al. 2013 [6] \\
\hline ERGIC1 rs1006721 & C vs. T & $0.69(0.58-0.83)$ & $6.22 \times 10^{-5}$ & GPA & Caucasian & $459 / 1503$ & 0.023 & 0.642 & 0.785 & 1.000 & 0.114 & 0.992 & 0.797 & 1.000 & Xie, et al. 2013 [6] \\
\hline OFCC1 rs9358619 & A vs. G & $1.45(1.21-1.74)$ & $4.90 \times 10^{-5}$ & GPA & Caucasian & $459 / 1503$ & 0.021 & 0.642 & 0.756 & 1.000 & 0.092 & 0.990 & 0.762 & 1.000 & Xie, et al. 2013 [6] \\
\hline
\end{tabular}


Table 4. Cont

\begin{tabular}{|c|c|c|c|c|c|c|c|c|c|c|c|c|c|c|c|}
\hline \multirow{3}{*}{ Gene/ Variant } & \multirow{3}{*}{ Comparison } & \multirow{3}{*}{ OR $(95 \% \mathrm{CI})$} & \multirow{3}{*}{$p$-Value } & \multirow{3}{*}{$\begin{array}{l}\text { Diagnosis } \\
\text { (Clinical/ } \\
\text { Serologic } \\
\text { Subtypes) }\end{array}$} & \multirow{3}{*}{ Ethnicity } & \multirow{3}{*}{$\begin{array}{c}\text { No. of } \\
\text { Cases/ } \\
\text { Controls }\end{array}$} & \multirow{3}{*}{$\begin{array}{l}\text { Power } \\
\text { OR } 1.2\end{array}$} & \multirow{3}{*}{$\begin{array}{l}\text { Power } \\
\text { OR } 1.5\end{array}$} & \multicolumn{4}{|c|}{ FPRP Values at Prior Probability } & \multirow{3}{*}{$\begin{array}{l}\text { BFDP } \\
0.001\end{array}$} & \multirow{3}{*}{$\begin{array}{l}\text { BFDP } \\
0.000001\end{array}$} & \multirow{3}{*}{ Author, Year } \\
\hline & & & & & & & & & \multicolumn{2}{|c|}{ OR 1.2} & \multicolumn{2}{|c|}{ OR 1.5} & & & \\
\hline & & & & & & & & & 0.001 & 0.000001 & 0.001 & 0.000001 & & & \\
\hline $\begin{array}{l}\text { HLA-DMA } \\
\text { rs3135029 }\end{array}$ & A vs. C & $1.61(1.28-2.02)$ & $4.55 \times 10^{-5}$ & GPA & Caucasian & $459 / 1503$ & 0.006 & 0.270 & 0.875 & 1.000 & 0.125 & 0.993 & 0.764 & 1.000 & Xie, et al. 2013 [6] \\
\hline $\begin{array}{l}\text { HLA-DOA } \\
\text { rs176248 }\end{array}$ & T vs. $C$ & $0.70(0.58-0.83)$ & $8.16 \times 10^{-5}$ & GPA & Caucasian & $459 / 1503$ & 0.022 & 0.713 & 0.644 & 0.999 & 0.054 & 0.983 & 0.668 & 1.000 & Xie, et al. 2013 [6] \\
\hline $\begin{array}{l}\text { HLA-DOA } \\
\text { rs206762 }\end{array}$ & C vs. T & $1.36(1.17-1.58)$ & $5.92 \times 10^{-5}$ & GPA & Caucasian & $459 / 1503$ & 0.051 & 0.900 & 0.534 & 0.999 & 0.061 & 0.985 & 0.703 & 1.000 & Xie, et al. 2013 [6] \\
\hline $\begin{array}{c}\text { HLA-DOA } \\
\text { rs9296068 }\end{array}$ & G vs. T & $0.68(0.58-0.81)$ & $7.49 \times 10^{-6}$ & GPA & Caucasian & $459 / 1503$ & 0.011 & 0.588 & 0.578 & 0.999 & 0.026 & 0.964 & 0.491 & 0.999 & Xie, et al. 2013 [6] \\
\hline $\begin{array}{l}\text { HLA-DPB2 } \\
\text { rs1810472 }\end{array}$ & G vs. A & $0.65(0.54-0.77)$ & $1.06 \times 10^{-6}$ & GPA & Caucasian & $459 / 1503$ & 0.002 & 0.385 & 0.235 & 0.997 & 0.002 & 0.618 & 0.062 & 0.985 & Xie, et al. 2013 [6] \\
\hline $\begin{array}{l}\text { HLA-DPB2 } \\
\text { rs3117035 }\end{array}$ & A vs. G & $0.72(0.61-0.84)$ & $2.50 \times 10^{-5}$ & GPA & Caucasian & $459 / 1503$ & 0.032 & 0.836 & 0.484 & 0.999 & 0.034 & 0.972 & 0.579 & 0.999 & Xie, et al. 2013 [6] \\
\hline $\begin{array}{l}\text { COL11A2 } \\
\text { rs2235498 }\end{array}$ & T vs. C & $0.67(0.55-0.81)$ & $3.86 \times 10^{-5}$ & GPA & Caucasian & $459 / 1503$ & 0.012 & 0.521 & 0.744 & 1.000 & 0.063 & 0.985 & 0.678 & 1.000 & Xie, et al. 2013 [6] \\
\hline WDR46 rs3130257 & T vs. C & $1.63(1.32-2.01)$ & $5.61 \times 10^{-6}$ & GPA & Caucasian & $459 / 1503$ & 0.002 & 0.218 & 0.700 & 1.000 & 0.022 & 0.957 & 0.365 & 0.998 & Xie, et al. 2013 [6] \\
\hline DAXX rs211474 & T vs. C & $0.71(0.60-0.84)$ & $6.37 \times 10^{-5}$ & GPA & Caucasian & $459 / 1503$ & 0.031 & 0.769 & 0.679 & 1.000 & 0.078 & 0.988 & 0.744 & 1.000 & Xie, et al. 2013 [6] \\
\hline KIFC1 rs211452 & Cvs. T & $0.65(0.55-0.76)$ & $1.98 \times 10^{-7}$ & GPA & Caucasian & $459 / 1503$ & 0.001 & 0.375 & 0.067 & 0.986 & 0.000 & 0.150 & 0.008 & 0.895 & Xie, et al. 2013 [6] \\
\hline $\begin{array}{l}\text { SYNGAP1 } \\
\text { rs211456 }\end{array}$ & A vs. C & $0.72(0.61-0.84)$ & $3.57 \times 10^{-5}$ & GPA & Caucasian & $459 / 1503$ & 0.032 & 0.836 & 0.484 & 0.999 & 0.034 & 0.972 & 0.579 & 0.999 & Xie, et al. 2013 [6] \\
\hline $\begin{array}{l}\text { SYNGAP1 } \\
\text { rs2247385 }\end{array}$ & G vs. A & $0.69(0.59-0.81)$ & $5.14 \times 10^{-6}$ & GPA & Caucasian & $459 / 1503$ & 0.011 & 0.663 & 0.353 & 0.998 & 0.009 & 0.896 & 0.271 & 0.997 & Xie, et al. 2013 [6] \\
\hline FLJ43752 rs210120 & G vs. A & $0.72(0.62-0.84)$ & $2.58 \times 10^{-5}$ & GPA & Caucasian & $459 / 1503$ & 0.032 & 0.836 & 0.484 & 0.999 & 0.034 & 0.972 & 0.579 & 0.999 & Xie, et al. 2013 [6] \\
\hline BCKDHB rs515347 & G vs. A & $1.70(1.32-2.18)$ & $3.13 \times 10^{-5}$ & GPA & Caucasian & $459 / 1503$ & 0.003 & 0.162 & 0.905 & 1.000 & 0.151 & 0.994 & 0.769 & 1.000 & Xie, et al. 2013 [6] \\
\hline TCBA1 rs6924068 & G vs. A & $1.40(1.20-1.63)$ & $1.37 \times 10^{-5}$ & GPA & Caucasian & $459 / 1503$ & 0.023 & 0.813 & 0.382 & 0.998 & 0.018 & 0.947 & 0.429 & 0.999 & Xie, et al. 2013 [6] \\
\hline MAGI2 rs3779312 & A vs. G & $1.50(1.26-1.78)$ & $5.26 \times 10^{-6}$ & GPA & Caucasian & $459 / 1503$ & 0.005 & 0.500 & 0.392 & 0.998 & 0.007 & 0.873 & 0.214 & 0.996 & Xie, et al. 2013 [6] \\
\hline CUTL1 rs1734729 & T vs. C & $1.41(1.20-1.67)$ & $4.54 \times 10^{-5}$ & GPA & Caucasian & $459 / 1503$ & 0.031 & 0.763 & 0.691 & 1.000 & 0.083 & 0.989 & 0.754 & 1.000 & Xie, et al. 2013 [6] \\
\hline DPP6 rs4726422 & G vs. A & $0.74(0.63-0.86)$ & $7.74 \times 10^{-5}$ & GPA & Caucasian & $459 / 1503$ & 0.061 & 0.913 & 0.586 & 0.999 & 0.086 & 0.989 & 0.766 & 1.000 & Xie, et al. 2013 [6] \\
\hline $\begin{array}{l}\text { LOC441376 } \\
\text { rs3019885 }\end{array}$ & G vs. T & $1.44(1.24-1.67)$ & $1.90 \times 10^{-6}$ & GPA & Caucasian & $459 / 1503$ & 0.008 & 0.705 & 0.151 & 0.994 & 0.002 & 0.667 & 0.092 & 0.990 & Xie, et al. 2013 [6] \\
\hline $\begin{array}{l}\text { SLC30A8 } \\
\text { rs1793729 }\end{array}$ & C vs. T & $0.68(0.57-0.80)$ & $5.68 \times 10^{-6}$ & GPA & Caucasian & $459 / 1503$ & 0.007 & 0.594 & 0.317 & 0.998 & 0.006 & 0.847 & 0.194 & 0.996 & Xie, et al. 2013 [6] \\
\hline $\begin{array}{l}\text { SLC30A8 } \\
\text { rs1695715 }\end{array}$ & T vs. C & $0.69(0.58-0.82)$ & $1.57 \times 10^{-5}$ & GPA & Caucasian & $459 / 1503$ & 0.016 & 0.652 & 0.610 & 0.999 & 0.037 & 0.975 & 0.582 & 0.999 & Xie, et al. 2013 [6] \\
\hline KCNK9 rs2447406 & T vs. C & $1.58(1.27-1.97)$ & $3.25 \times 10^{-5}$ & GPA & Caucasian & $459 / 1503$ & 0.007 & 0.322 & 0.869 & 1.000 & 0.130 & 0.993 & 0.781 & 1.000 & Xie, et al. 2013 [6] \\
\hline C9orf66 rs584922 & T vs. C & $0.70(0.60-0.82)$ & $1.30 \times 10^{-5}$ & GPA & Caucasian & $459 / 1503$ & 0.015 & 0.727 & 0.392 & 0.998 & 0.013 & 0.932 & 0.365 & 0.998 & Xie, et al. 2013 [6] \\
\hline C9orf93 rs1341740 & T vs. C & $1.52(1.23-1.87)$ & $9.73 \times 10^{-5}$ & GPA & Caucasian & $459 / 1503$ & 0.013 & 0.450 & 0.855 & 1.000 & 0.142 & 0.994 & 0.814 & 1.000 & Xie, et al. 2013 [6] \\
\hline $\begin{array}{l}\text { LRRN6C } \\
\text { rs10491888 }\end{array}$ & G vs. A & $1.54(1.25-1.91)$ & $5.75 \times 10^{-5}$ & GPA & Caucasian & $459 / 1503$ & 0.012 & 0.405 & 0.880 & 1.000 & 0.173 & 0.995 & 0.837 & 1.000 & Xie, et al. 2013 [6] \\
\hline PAEP rs705669 & G vs. A & $0.69(0.57-0.83)$ & $8.53 \times 10^{-5}$ & GPA & Caucasian & $459 / 1503$ & 0.023 & 0.642 & 0.785 & 1.000 & 0.114 & 0.992 & 0.797 & 1.000 & Xie, et al. 2013 [6] \\
\hline $\begin{array}{c}\text { NEUROG3 } \\
\text { rs731573 }\end{array}$ & T vs. C & $1.44(1.20-1.72)$ & $9.40 \times 10^{-5}$ & GPA & Caucasian & $459 / 1503$ & 0.022 & 0.674 & 0.722 & 1.000 & 0.079 & 0.988 & 0.738 & 1.000 & Xie, et al. 2013 [6] \\
\hline TMPO rs2216021 & C vs. T & $1.36(1.17-1.57)$ & $6.14 \times 10^{-5}$ & GPA & Caucasian & $459 / 1503$ & 0.044 & 0.909 & 0.382 & 0.998 & 0.029 & 0.967 & 0.544 & 0.999 & Xie, et al. 2013 [6] \\
\hline TMPO rs2011247 & C vs. T & $0.73(0.63-0.85)$ & $4.84 \times 10^{-5}$ & GPA & Caucasian & $459 / 1503$ & 0.044 & 0.879 & 0.534 & 0.999 & 0.054 & 0.983 & 0.680 & 1.000 & Xie, et al. 2013 [6] \\
\hline FGF9 rs2031421 & T vs. G & $1.44(1.21-1.72)$ & $4.01 \times 10^{-5}$ & GPA & Caucasian & $459 / 1503$ & 0.022 & 0.674 & 0.722 & 1.000 & 0.079 & 0.988 & 0.738 & 1.000 & Xie, et al. 2013 [6] \\
\hline DOK5 rs6023640 & T vs. G & $1.40(1.18-1.66)$ & $8.98 \times 10^{-5}$ & GPA & Caucasian & $459 / 1503$ & 0.038 & 0.786 & 0.740 & 1.000 & 0.121 & 0.993 & 0.815 & 1.000 & Xie, et al. 2013 [6] \\
\hline PDE9A rs2269127 & A vs. $G$ & $1.65(1.36-2.01)$ & $4.06 \times 10^{-7}$ & GPA & Caucasian & $459 / 1503$ & 0.001 & 0.172 & 0.457 & 0.999 & 0.004 & 0.793 & 0.094 & 0.990 & Xie, et al. 2013 [6] \\
\hline
\end{tabular}




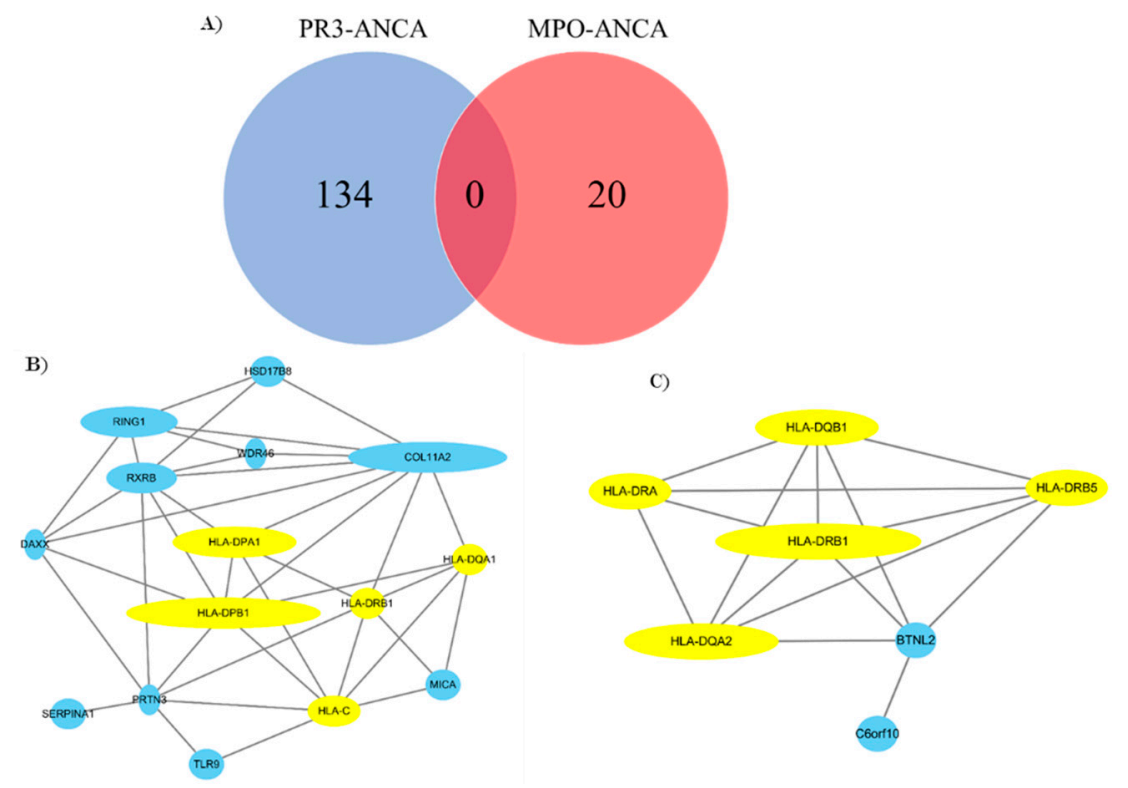

Figure 2. The number of noteworthy SNPs in PR3-ANCA and MPO-ANCA and their protein-protein networks (PPIs). (A) The overlap of SNPs in PR3-ANCA and MPO-ANCA; the lack of any shared SNPs between these two subtypes of ANCA-associated vasculitis. (B) PPI network of genes associated with PR3-ANCA vasculitis. (C) PPI network of genes associated with MPO-ANCA vasculitis. Borderline SNPs $\left(5.0 \times 10^{-8}<p<0.05\right)$ from GWASs that have been noteworthy in our analysis were also included. The yellow nodes represent the genes associated with the interferon-gamma-mediated signaling pathway. The number of SNPs associated with PR3-ANCA and MPO-ANCA is 134 and 20, respectively, which leads to a total of 21 and 8 unique genes. Note that some genes have multiple SNPs associated with PR3-ANCA and MPO-ANCA. Among these genes, only 15 genes and 7 genes are included in the PPI network by revealing known PPI interactions in Figure 2B,C.

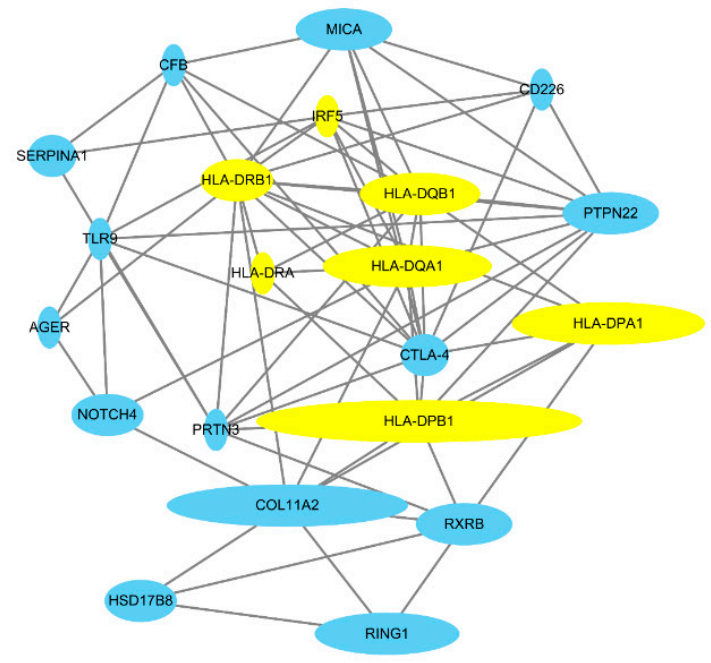

Figure 3. Protein-protein interaction network of associated genes in the etiopathogenesis of ANCA-associated vasculitis. The size of the node indicates the number of noteworthy SNPs of each gene. This network includes noteworthy SNPs and GWAS SNPs. The variants are listed in Table S7. The yellow nodes represent the genes associated with the interferon-gamma-mediated signaling pathway.

To investigate disease pathways, gene ontology (GO) enrichment analysis was performed. Several GO terms enriched in the SNPs set were identified by GO enrichment analysis for each subtype. Immune-related GO terms, including "antigen processing and presentation of peptide or polysaccharide antigen via MHC class II" (GO:0002504) with a $p$-value of $4.4 \times 10^{-17}$, 
"interferon-gamma-mediated (IFN- $\gamma$ ) signaling pathway" (GO:0060333) with a $p$-value of $2.9 \times 10^{-12}$, and "T cell receptor signaling pathway" (GO:0050852) with a $p$-value of $5.5 \times 10^{-10}$, were identified as the most prominently enriched AAV-related gene sets (Figure 4 and Figure S3)

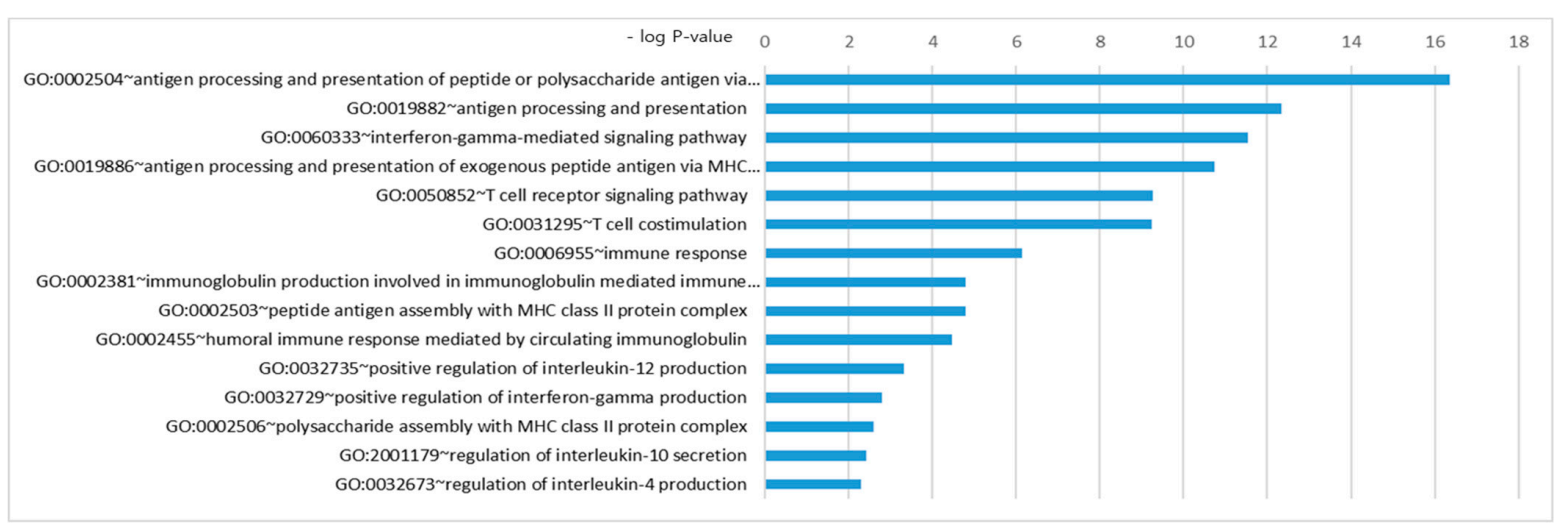

Figure 4. Gene ontology enrichment analysis of ANCA-associated vasculitis. The analysis was performed with the associated genetic variants. The cut-off p-value for this analysis was 0.01 $(-\log p$-value $=2)$. Other subtype results are shown in the Figure S3.

\subsection{Bullets}

1. Several studies in ANCA-associated vasculitis have reported statistically significant genetic variants.

2. Integration and further analysis of the data from several studies using FPRP, BFDP, and GO analysis identified novel biological pathways in ANCA-associated vasculitis, including a role of the IFN- $\gamma$ pathway.

3. Our results underline that there is a dichotomy between the respective target antigens, PR3 and MPO.

\section{Discussion}

In this study we investigated whether the genetic variants previously found to be associated with AAV are truly associated with AAV or are false-positives. Former studies have demonstrated that the target antigens of AAV, MPO, and PR3 are better discriminators of the disease than the clinical phenotypes, MPA and GPA [2]. Meta-analyses and GWASs have confirmed a genetic background of AAV. Our analysis found no genetic overlap of MPO-ANCA and PR3-ANCA vasculitis. This further underlines the need to distinguish both subtypes in clinical studies and may lead to a re-classification of $\mathrm{AAV}$ based on the serotypes. Our analyses further highlight that AAV is not a multigenic disease with the implication of several genetic variants contributing to one's individual risk to develop vasculitis.

This work used FPRP and BFDP estimation to re-analyze genetic associations from the retrieved research papers. Discovered SNPs of each included study with $p$-values under 0.05 or either $5.0 \times 10^{-8}$ were computed by both methods. Nearly half of the significant SNPs reported in meta-analyses of the observational studies were not noteworthy, whereas those SNPs in GWASs and their meta-analyses were noteworthy with FPRP and BFDP. However, when we applied the Bayesian procedures to SNPs associated with borderline error probabilities $\left(5.0 \times 10^{-8}<p\right.$-value $\left.<0.05\right)$ observed in a GWAS including patients with GPA, FPRP and BFDP computation yielded a noteworthiness of $85 \%$ of these SNPs.

The exact mechanisms leading to AAV onset are unclear, but a genetic predisposition and self-intolerance to environmental exposures, such as infections, have been proposed [14]. A current pathogenetic model proposes the initiation of an inflammatory cascade with the release of PR3 (pro-inflammatory cytokine) by an unknown insult, further selecting PR3-specific B lymphocytes producing ANCA and PR3 specific T cells [14]. Subsequently, neutrophils are activated by ANCA priming, leading to the destruction of endothelial cells by firmly attached neutrophils on endothelial 
cells $[15,16]$. Our GO enrichment analysis showed that the processing of antigens via MHC class II, the IFN- $\gamma$-mediated pathway and the $\mathrm{T}$ cell receptor signaling pathway are the main biological processes disturbed in AAV. GO enrichment analysis identified a crucial role of the IFN- $\gamma$ mediated signaling pathway and showed comparable involvement in both, MPO-ANCA and PR3-ANCA vasculitis. PPI analysis revealed a role of the interferon regulatory factor 5 (IRF5) in the model including patients with GPA and AAV. In systemic lupus erythematosus (SLE), IRF5 genetic variants are associated with an increased risk to develop the disease, alongside elevation of IRF5 expression and IFN production [17]. IFN- $\gamma$ has been studied in two biomarker studies involving a cohort with AAV and one cohort of patients with GPA. Analysis of samples obtained from patients with AAV recruited to participate in the RAVE trial indicated significant higher levels of IFN $-\gamma$ during active disease compared to remission (137 samples each) and to healthy controls (68 samples) [18]. Furthermore, the expression of chemokine (C-X-C motif) ligand (CXCL10 or IFN- $\gamma$-induced protein 10) was tested in the same study. CXCL10 expression is dependent on IFN- $\gamma$ and is implicated in several autoimmune disorders [19]. In patients with AAV, CXCL10 levels did not differ among patients with active disease or in remission, while CXCL10 levels were significantly lower in healthy controls. This argues for a sufficient reduction of IFN- $\gamma$ once remission is achieved in AAV, but in contrast, the levels of CXCL10 remain elevated. More research is clearly needed to understand the regulation of the IFN- $\gamma$ pathway in AAV.

IFN- $\gamma$ increases MHC class II expression in antigen-presenting cells (B cells, macrophages, and dendritic cells) that are engaged by microbial invasions [20,21]. Furthermore, IFN- $\gamma$ negatively regulates anti-inflammatory IL-10 production and induces pro-inflammatory IL-12 secretion [22]. IL-12 drives helper T(Th) cells to differentiate towards a Th1 phenotype and stimulates natural killer (NK) cells to produce IFN- $\gamma$. IFN- $\gamma$ secreted from NK cells induces monocyte differentiation towards active macrophages in the local inflammatory site [23]. In addition, MPO stimulation leads to a dose-dependent production of IFN- $\gamma$ by monocytes [24]. MPO induces the formation of neutrophil extracellular traps (NET), which is thought to be a crucial factor in the cascade of inflammation in AAV [25]. The IFN- $\gamma$ pathway may play a role in the development of local inflammation. Alongside the immune system activation and NET formation as crucial mechanisms in the inflammatory processes, our data confirm that genetic predisposition and the activation of antigen-presenting cells with the selection of autoimmune T cells are important in the pathogenesis of AAV.

Our PPI network shows the mainframe structure of genetic interactions among HLA-associated genes and COL11A2 in GPA, PR3-ANCA vasculitis, and AAV itself. COL11A2 gene codes for the pro- $\alpha 2$ chain of collagen type XI. COL11A2 was demonstrated to be in linkage disequilibrium with the HLA-DP gene in an AAV GWAS $[3,26]$. Except MHC coding genes, other genetic risk factors associated with AAV were PTPN22, RXRB, CTLA-4, MICA, SERPINA1, PRTN3, CD226, TLR9, IRF5, NOTCH4, AGER, and CFB, which is consistent with previous findings (Figure 3). PTPN22 encodes protein tyrosine phosphatase in lymphoid tissues with an abnormal regulatory CD4 T-cell (Treg) function and increased neutrophil function reported in PTPN22 variant (rs2476601) [27]. RXRB is a gene encoding a family of retinoid $\mathrm{X}$ receptors, which form homodimers and heterodimers with retinoic acid, thyroid hormone, and vitamin D receptors [28]. There was a functional difference in the responsiveness to vitamin A and vitamin D in AAV patients, which could not be explained by RXRB polymorphisms alone [29]. CTLA-4 is a gene encoding an inhibitory surface protein on activated T cells that interacts with CD80 or CD86 and competes with CD28 (co-stimulatory molecule) [30]. Abatacept, a selective modulator of the CD80/86-CD28 costimulatory signal was successfully used in patients with non-severe GPA and is currently in a phase III trial (ABROGATE, NCT02108860), which suggests that the CTLA-4 protein might be of importance in AAV pathogenesis [31]. SERPINA1 and PRTN3 are also referred to as crucial genes in the pathogenesis of PR3-ANCA vasculitis [27]. PRTN3 is the gene encoding PR3 that exists on the surface or in the cytoplasm of neutrophils, whereas SERPINA1 encodes $\alpha-1$ antitrypsin, the major inhibitory molecule of PR3. Previous findings suggest that PRTN3 genetic variants determine the predominant location of PR3 in neutrophils, where it may affect the activity of 
neutrophils [27]. No noteworthy variants were observed in MPA, but genetic variants related to the HLA-DR and HLA-DQ loci were found to be associated with MPO-ANCA vasculitis (Figure S2).

FPRP was criticized with respect to its heuristic derivation of the formula, especially with the use of $\alpha$ and $1-\beta$ as the probabilities of observing values greater or less than the test quantities under a null and alternative hypothesis [32]. Even though a labored statistical derivation exists, the concept of "noteworthiness" originated from FPRP has influenced recent genetic studies [12]. Moreover, although direct comparison between BFDP and FPRP is not possible, the behavior of both is similar in promoting SNPs' rankings [13]. BFDP produced more noteworthy findings than FPRP because the latter derives smaller posterior null estimates due to the conditioning on tail areas unlike the reliance on point estimates provided by BFDP $[13,33]$.

Our study has some limitations. First, different GWASs included in this study share the same cohorts. Therefore, slight overestimation of some sharing genes found in these studies may exist. However, no effect will be present in the gene network analysis because weighting of a gene corresponded to the number of significant SNPs in each gene. Second, we were not able to analyze variants with incomplete data (those without $95 \%$ CIs). Thus, it was not possible to confirm that all genome-wide significant SNPs satisfied the FPRP and BFDP thresholds. In fact, SNPs significant in GWASs were included in GO enrichment analysis, suggesting that these SNPs would be noteworthy due to previous results (Tables 4 and 4 and Tables S5 and S6). Furthermore, the low frequency of patients with MPA in the included studies may have led to the finding of no noteworthy SNPs in our re-analysis. Lastly, linkage disequilibrium and expression changes of SNPs were not considered in this analysis, perhaps treating SNPs and its properties too simply. Further research comprising quantitative expression of loci is needed.

Nevertheless, our study merged current results of genetic associations in AAV and its subtypes. In addition, the investigation of false-positive results in genetic research proves that researchers should pay careful attention when interpreting the positive results reported in previous papers. All GWAS SNPs associated with a borderline $p$-value are worth further examination with various statistical methods, as we found many of them to be noteworthy with the used Bayesian methods. Moreover, we highlight the importance of the IFN- $\gamma$ pathway in the pathogenesis of AAV through GO enrichment analysis using GWAS and meta-analysis gene sets. This suggests that further research into the IFN- $\gamma$ pathway in AAV may lead to the development of novel therapeutic approaches for this complex disease.

Supplementary Materials: The following are available online at http://www.mdpi.com/2077-0383/8/2/266/s1. Figure S1: The Venn diagram for all subtypes and AAV with the noteworthy SNPs from our FPRP and BFDP analyses. Figure S2: Protein-protein interaction network of GPA (A), MPO-ANCA vasculitis (B), PR3-ANCA vasculitis (C), and ANCA-associated vasculitis (D). Genes are weighted according to the number of the SNPs of each gene. The gene set was composed of GWAS discovered SNP genes and noteworthy SNP genes in the meta-analyses of observational studies and GWAS meta-analyses. Borderline SNPs $\left(5.0 \times 10^{-8}<p<0.05\right)$ from GWASs that are noteworthy were also included in this analysis. Figure S3. Gene ontology enrichment analysis of GPA (A), MPO-ANCA vasculitis (B), PR3-ANCA vasculitis (C), and ANCA-associated vasculitis (D). The gene set was composed of GWAS discovered SNP genes and noteworthy SNP genes in the meta-analyses of observational studies and GWAS meta-analyses. Borderline SNPs $\left(5.0 \times 10^{-8}<p<0.05\right)$ from GWASs that are noteworthy were also included in this analysis. Table S1: Preferred Reporting Items for Systematic reviews and Meta-Analyses (PRISMA) checklist. Table S2. List of publications included in this re-analysis. Table S3. Meta-analysis results of observational studies, gene variants with statistical significance $(p$-value $<0.05)$ for each clinical diagnosis (AAV, GPA, and MPA). Summary of genetic variants with FPRP and BFDP values, and noteworthy genetic variants are shown in bold. Table S4. Meta-analysis results of observational studies; gene variants with statistical significance $(p$-value $<0.05)$ for each serologic diagnosis (MPO-ANCA, PR3-ANCA). Summary of genetic variants with FPRP and BFDP values, and noteworthy genetic variants are shown in bold. Table S5. Results of meta analyses (combined analysis) with genome-wide association studies and replication cohort. Noteworthy genetic variants with satisfied FPRP and BFDP values are shown in bold. Table S6. Re-analysis of the SNPs discovered in genome-wide association studies of patients with GPA. Xie et al. only provided GPA SNPs with ORs and 95\% CIs. Table S7: These gene sets were computed in gene ontology enrichment analysis and protein-protein interaction network analysis. This gene set include noteworthy variants from meta-analyses and statistically significant GWAS SNPs. Equation S1: Calculation of false positive report probability (FPRP) and Bayesian false discovery probability (BFDP). 
Author Contributions: Conceptualization, K.S.L., A.K., and J.I.S.; formal analysis, K.S.L. and Y.K.; investigation, K.S.L. and Y.S.O.; data curation, K.S.L. and Y.S.O.; writing-original draft preparation, K.S.L., D.F.P.V., F.R.P.d.S., and Y.K.; writing-review and editing, A.K., M.E., P.A.M., D.J., C.I.A., K.A.S., C.R., K.H.L., and J.I.S.; supervision, J.I.S.; project administration, J.I.S.

Funding: KAS is supported by a Canada Research Chair and holds the Sherman Chair in Genomic Medicine. The GWASs assessed were supported by the Erna Baird Memorial Grant and Vasculitis Foundation Canada. The GWASs of the Vasculitis Clinical Research Consortium (VCRC) were supported by the US NIH (National Institute of Arthritis and Musculoskeletal and Skin Diseases grants U54-AR-057319 and R01-AR-047799, National Center for Research Resources grant U54-RR-019497, the Office of Rare Diseases Research, and the National Center for Advancing Translational Sciences). The VCRC is part of the Rare Diseases Clinical Research Network.

Conflicts of Interest: The authors declare no conflict of interest. The funders had no role in the design of the study; in the collection, analyses, or interpretation of data; in the writing of the manuscript, or in the decision to publish the results.

\section{References}

1. Pagnoux, C. Updates in anca-associated vasculitis. Eur. J. Rheumatol. 2016, 3, 122-133. [CrossRef] [PubMed]

2. Mahr, A.; Katsahian, S.; Varet, H.; Guillevin, L.; Hagen, E.C.; Hoglund, P.; Merkel, P.A.; Pagnoux, C.; Rasmussen, N.; Westman, K.; et al. Revisiting the classification of clinical phenotypes of anti-neutrophil cytoplasmic antibody-associated vasculitis: A cluster analysis. Ann. Rheum. Dis. 2013, 72, 1003-1010. [CrossRef] [PubMed]

3. Lyons, P.A.; Rayner, T.F.; Trivedi, S.; Holle, J.U.; Watts, R.A.; Jayne, D.R.; Baslund, B.; Brenchley, P.; Bruchfeld, A.; Chaudhry, A.N.; et al. Genetically distinct subsets within anca-associated vasculitis. N. Engl. J. Med. 2012, 367, 214-223. [CrossRef] [PubMed]

4. Merkel, P.A.; Xie, G.; Monach, P.A.; Ji, X.; Ciavatta, D.J.; Byun, J.; Pinder, B.D.; Zhao, A.; Zhang, J.; Tadesse, Y.; et al. Identification of functional and expression polymorphisms associated with risk for antineutrophil cytoplasmic autoantibody-associated vasculitis. Arthritis Rheumatol. 2017, 69, 1054-1066. [CrossRef] [PubMed]

5. Rahmattulla, C.; Mooyaart, A.L.; van Hooven, D.; Schoones, J.W.; Bruijn, J.A.; Dekkers, O.M.; Bajema, I.M. Genetic variants in anca-associated vasculitis: A meta-analysis. Ann. Rheum. Dis. 2016, 75, 1687-1692. [CrossRef] [PubMed]

6. Xie, G.; Roshandel, D.; Sherva, R.; Monach, P.A.; Lu, E.Y.; Kung, T.; Carrington, K.; Zhang, S.S.; Pulit, S.L.; Ripke, S.; et al. Association of granulomatosis with polyangiitis (Wegener's) with HLA-DPB1*04 and SEMA6A gene variants: Evidence from genome-wide analysis. Arthritis Rheum. 2013, 65, 2457-2468. [CrossRef]

7. Moher, D.; Liberati, A.; Tetzlaff, J.; Altman, D.G.; Group, P. Preferred reporting items for systematic reviews and meta-analyses: The prisma statement. BMJ 2009, 339, 2535. [CrossRef]

8. Lee, Y.H.; Choi, S.J.; Ji, J.D.; Song, G.G. CTLA-4 and TNF-alpha promoter-308 A/G polymorphisms and ANCA-associated vasculitis susceptibility: A meta-analysis. Mol. Biol. Rep. 2012, 39, 319-326. [CrossRef]

9. Jung, J.H.; Song, G.G.; Lee, Y.H. Meta-analysis of associations between interleukin-10 polymorphisms and susceptibility to vasculitis. Immunol. Investig. 2015, 44, 553-565. [CrossRef]

10. Chung, S.A.; Xie, G.; Roshandel, D.; Sherva, R.; Edberg, J.C.; Kravitz, M.; Dellaripa, P.F.; Hoffman, G.S.; Mahr, A.D.; Seo, P.; et al. Meta-analysis of genetic polymorphisms in granulomatosis with polyangiitis (Wegener's) reveals shared susceptibility loci with rheumatoid arthritis. Arthritis Rheum. 2012, 64, 3463-3471. [CrossRef]

11. Lee, Y.H.; Choi, S.J.; Ji, J.D.; Song, G.G. The protein tyrosine phosphatase nonreceptor 22 C1858T polymorphism and vasculitis: A meta-analysis. Mol. Biol. Rep. 2012, 39, 8505-8511. [CrossRef] [PubMed]

12. Wacholder, S.; Chanock, S.; Garcia-Closas, M.; El Ghormli, L.; Rothman, N. Assessing the probability that a positive report is false: An approach for molecular epidemiology studies. J. Natl. Cancer Inst. 2004, 96, 434-442. [CrossRef] [PubMed]

13. Wakefield, J. A bayesian measure of the probability of false discovery in genetic epidemiology studies. Am. J. Hum. Genet. 2007, 81, 208-227. [CrossRef] [PubMed]

14. Cartin-Ceba, R.; Peikert, T.; Specks, U. Pathogenesis of anca-associated vasculitis. Curr. Rheumatol. Rep. 2012, 14, 481-493. [CrossRef] [PubMed]

15. Brogan, P.; Eleftheriou, D. Vasculitis update: Pathogenesis and biomarkers. Pediatr. Nephrol. 2018, 33, $187-198$. [CrossRef] [PubMed] 
16. Jarrot, P.A.; Kaplanski, G. Pathogenesis of anca-associated vasculitis: An update. Autoimmun. Rev. 2016, 15, 704-713. [CrossRef] [PubMed]

17. Barnes, B.J. Genetic versus non-genetic drivers of SLE: Implications of IRF5 dysregulation in both roads leading to SLE. Curr. Rheumatol. Rep. 2019, 21, 2. [CrossRef]

18. Monach, P.A.; Warner, R.L.; Tomasson, G.; Specks, U.; Stone, J.H.; Ding, L.; Fervenza, F.C.; Fessler, B.J.; Hoffman, G.S.; Ikle, D.; et al. Serum proteins reflecting inflammation, injury and repair as biomarkers of disease activity in anca-associated vasculitis. Ann. Rheum. Dis. 2013, 72, 1342-1350. [CrossRef]

19. Antonelli, A.; Ferrari, S.M.; Giuggioli, D.; Ferrannini, E.; Ferri, C.; Fallahi, P. Chemokine (C-X-C motif) ligand (CXCL)10 in autoimmune diseases. Autoimmun. Rev. 2014, 13, 272-280. [CrossRef]

20. Nathan, C.F.; Prendergast, T.J.; Wiebe, M.E.; Stanley, E.R.; Platzer, E.; Remold, H.G.; Welte, K.; Rubin, B.Y.; Murray, H.W. Activation of human macrophages. Comparison of other cytokines with interferon-gamma. J. Exp. Med. 1984, 160, 600-605. [CrossRef]

21. Steimle, V.; Siegrist, C.A.; Mottet, A.; Lisowska-Grospierre, B.; Mach, B. Regulation of MHC class II expression by interferon-gamma mediated by the transactivator gene CIITA. Science 1994, 265, 106-109. [CrossRef] [PubMed]

22. Wilke, C.M.; Wei, S.; Wang, L.; Kryczek, I.; Kao, J.; Zou, W. Dual biological effects of the cytokines interleukin-10 and interferon-gamma. Cancer Immunol. Immunother. 2011, 60, 1529-1541. [CrossRef] [PubMed]

23. Goldszmid, R.S.; Caspar, P.; Rivollier, A.; White, S.; Dzutsev, A.; Hieny, S.; Kelsall, B.; Trinchieri, G.; Sher, A. Nk cell-derived interferon-gamma orchestrates cellular dynamics and the differentiation of monocytes into dendritic cells at the site of infection. Immunity 2012, 36, 1047-1059. [CrossRef] [PubMed]

24. Yamaguchi, R.; Kawata, J.; Yamamoto, T.; Ishimaru, Y.; Sakamoto, A.; Ono, T.; Narahara, S.; Sugiuchi, H.; Hirose, E.; Yamaguchi, Y. Mechanism of interferon-gamma production by monocytes stimulated with myeloperoxidase and neutrophil extracellular traps. Blood Cells Mol. Dis. 2015, 55, 127-133. [CrossRef] [PubMed]

25. Metzler, K.D.; Fuchs, T.A.; Nauseef, W.M.; Reumaux, D.; Roesler, J.; Schulze, I.; Wahn, V.; Papayannopoulos, V.; Zychlinsky, A. Myeloperoxidase is required for neutrophil extracellular trap formation: Implications for innate immunity. Blood 2011, 117, 953-959. [CrossRef] [PubMed]

26. Relle, M.; Fohr, B.; Fasola, F.; Schwarting, A. Genetics and pathophysiology of granulomatosis with polyangiitis (GPA) and its main autoantigen proteinase 3. Mol. Cell Probes 2016, 30, 366-373. [CrossRef]

27. Alberici, F.; Martorana, D.; Vaglio, A. Genetic aspects of anti-neutrophil cytoplasmic antibody-associated vasculitis. Nephrol. Dial. Transplant. 2015, 30, 37-45. [CrossRef]

28. Yu, V.C.; Delsert, C.; Andersen, B.; Holloway, J.M.; Devary, O.V.; Naar, A.M.; Kim, S.Y.; Boutin, J.M.; Glass, C.K.; Rosenfeld, M.G. RXR beta: A coregulator that enhances binding of retinoic acid, thyroid hormone, and vitamin D receptors to their cognate response elements. Cell 1991, 67, 1251-1266. [CrossRef]

29. Kalsch, A.I.; Peters, A.; Buhl, B.; Breedijk, A.; Prem, K.; Schmitt, W.H.; Weiss, C.; Heeringa, P.; Kallenberg, C.; Birck, R.; et al. Retinoid x receptor beta polymorphisms do not explain functional differences in vitamins d and a response in antineutrophil cytoplasmic antibody associated vasculitis patients. Autoimmunity 2009, 42, 467-474. [CrossRef]

30. Noel, P.J.; Boise, L.H.; Green, J.M.; Thompson, C.B. CD28 costimulation prevents cell death during primary T cell activation. J. Immunol. 1996, 157, 636-642.

31. Langford, C.A.; Monach, P.A.; Specks, U.; Seo, P.; Cuthbertson, D.; McAlear, C.A.; Ytterberg, S.R.; Hoffman, G.S.; Krischer, J.P.; Merkel, P.A.; et al. An open-label trial of abatacept (CTLA4-IG) in non-severe relapsing granulomatosis with polyangiitis (Wegener's). Ann. Rheum. Dis. 2014, 73, 1376-1379. [CrossRef] [PubMed]

32. Lucke, J.F. A critique of the false-positive report probability. Genet. Epidemiol. 2009, 33, 145-150. [CrossRef] [PubMed]

33. Wakefield, J. Reporting and interpretation in genome-wide association studies. Int. J. Epidemiol. 2008, 37, 641-653. [CrossRef] [PubMed]

(C) 2019 by the authors. Licensee MDPI, Basel, Switzerland. This article is an open access article distributed under the terms and conditions of the Creative Commons Attribution (CC BY) license (http:/ / creativecommons.org/licenses/by/4.0/). 